\title{
Numerical simulation of solar photospheric jet-like phenomena caused by magnetic
}

\section{reconnection}

\author{
Yuji KOTANI ${ }^{1}$ and Kazunari SHIBATA ${ }^{2}$ \\ ${ }^{1}$ Department of Astronomy, Graduate School of Science, Kyoto University, \\ Kitashirakawa-Oiwake-cho, Sakyo-ku, Kyoto, Kyoto 606-8502, Japan \\ ${ }^{2}$ Kwasan and Hida Observatories, Kyoto University, 17 Ohmine-cho Kita Kazan, \\ Yamashina-ku, Kyoto, Kyoto 607-8471, Japan \\ *E-mail: kotani@kusastro.kyoto-u.ac.jp \\ Received $\langle 2020$ March 10 ); Accepted 2020 June 19〉
}

\begin{abstract}
Jet phenomena with a bright loop in their footpoint, called anemone jets, have been observed in the solar corona and chromosphere. These jets are formed as a consequence of magnetic reconnection, and from the scale universality of magnetohydrodynamics (MHD), it can be expected that anemone jets exist even in the solar photosphere. However, it is not necessarily apparent that jets can be generated as a result of magnetic reconnection in the photosphere, where the magnetic energy is not dominant. Furthermore, MHD waves generated from the photospheric jets could contribute to chromospheric heating and spicule formation; however, this hypothesis has not yet been thoroughly investigated. In this study, we perform 3D MHD simulation including gravity with the solar photospheric parameter to investigate anemone jets in the solar photosphere. In the simulation, jet-like structures were induced by magnetic reconnection in the solar photosphere. We determined that these jet-like structures were caused by slow shocks formed by the reconnection and were propagated approximately in the direction of the background magnetic field. We also suggested that MHD waves from the jet-like structures could influence local atmospheric heating and spicule formation.
\end{abstract}


Key words: magnetic reconnection — Sun: photosphere - magnetohydrodynamics (MHD)

\section{Introduction}

In the solar atmosphere, different kinds of jet phenomena, that is, the ejection of collimated plasma, can be observed at different wavelengths. In particular, numerous jets with a bright loop at their footpoint can be observed. These jets are called inverted-Y, Eiffel tower-shaped, or anemone jets because of their appearance (e.g., Shibata et al. 1994; Shibata et al. 2007; Nisticò et al. 2009). Herein, we use the term anemone jets" to refer to these jets in this paper.

Anemone jets were first discovered in the corona by the Yohkoh satellite (Shibata et al. 1992; Shimojo et al. 1996). Shibata (1999) proposed that these jets are formed as a consequence of magnetic reconnection similar to large-scale flares followed by coronal mass ejections. Today, numerous simulations have been performed in two-dimensional (2D) (Yokoyama \& Shibata 1996) and three-dimensional (3D) scenarios (Moreno-Insertis et al. 2008, 2013; Török et al. 2009; Pariat et al. 2009, 2015a, 2016; Archontis \& Hood 2013; Fang et al. 2014; Lee et al. 2015; Wyper et al. 2018) and their results support this theory.

Approximately ten years after the Yohkoh satellite discovery, small anemone jets were found in the chromosphere by observation with the Hinode satellite (Shibata et al. 2007; Nishizuka et al. 2008; Singh et al. 2011). Although the spatial scale and velocity of the anemone jets differ significantly between the corona and chromosphere (see table 1), their observational features and numerical simulations (Nishizuka et al. 2008; Takasao et al. 2013) support the fact that chromospheric anemone jets are also explained by magnetic reconnection. In both coronal and chromospheric cases, the jets speed is approximately the Alfvén speed, and the lifetime normalized by the Alfvén time is approximately $10-100$. These properties are consistent with reconnection theory and suggest that anemone jets are induced by reconnection regardless of their spatial scale. This fact reflects the scale universality of magnetohydrodynamics (MHD).

From the MHD scale universality, it can be expected that anemone jets having physical values as indicted in table 1 occur in the solar photosphere. However, considering that the solar photosphere is not magnetically dominant (plasma beta $\beta \sim 1-10$ ), it is not apparent that jets can be created as a result of magnetic reconnection, as in the chromosphere and corona of $\beta \ll 1$. 
Pariat et al. (2016) performed 3D MHD simulation in an environment of $\beta \simeq 1$; however, this calculation did not include gravity, and hence it could possibly not apply to the dynamics in the photosphere. Moreover, owing to the lack of spatial resolution, observing jets with a size of approximately $100 \mathrm{~km}$ remains a challenge. Several cases of brightening with inverted-Y-shaped loops in the upper photosphere and the lower chromosphere have been reported (Yurchyshyn et al. 2011; Bharti et al. 2017; Chitta et al. 2017; Tian et al. 2018; Nelson et al. 2019); however, their length is approximately $1000 \mathrm{~km}$. The jet phenomenon of approximately $100 \mathrm{~km}$ has not yet been observed.

The presence of photospheric anemone jets could be valuable as a source of waves in the lower solar atmosphere. When a photospheric anemone jet is generated, the surrounding pressure and magnetic field fluctuate and MHD waves are generated. Because the solar atmosphere is stratified by gravity, these MHD waves propagate to the upper layer with growing amplitude and form shock waves, which could drive spicules and surges. Such a growth process of MHD waves is the same mechanism that drives spicules by p-mode leakage and slow mode MHD waves generated from nonlinear Alfvén waves (Suematsu et al. 1982; De Pontieu et al. 2004; Hollweg et al. 1982; Kudoh, \& Shibata 1999; Wang \& Yokoyama 2020) or surges from Ellerman bombs (e.g., Yang et al. 2014). As a model to explain the spicule formation with the same mechanism as coronal jets, Sterling \& Moore (2016) proposed that spicules are formed by the ejection of microfilament. However, if the microfilament ejected at Alfvén speed is not re-accelerated, the height that can be achieved is $\sim H / \beta(H$ : scale height $\sim 150 \mathrm{~km}$ in the solar photosphere). Therefore, it is difficult for the microfilament itself to achieve the height of the spicule even if the microfilament erupts from the photosphere or lower chromosphere with $\beta \sim 1$. Hence, even when considering microfilament ejection, it is necessary to consider the MHD waves generated by the ejection or the re-acceleration mechanism of the microfilament. Moreover, magnetic reconnection has been proposed as the origin of the waves necessary to cause chromospheric and coronal heating (e.g., Parker 1991). If reconnection events in the lower atmosphere provide waves to heat, photospheric jets could be a candidate. For these reasons, we must study photospheric anemone jets to determine spicule models and clarify the heating mechanism of the solar atmosphere.

In this study, we perform a 3D MHD simulation of magnetic reconnection with solar photospheric parameters to investigate the properties of photospheric anemone jets. Then, we discuss the degree to which MHD waves are generated from the jets and how they influence the upper atmosphere. 
Table 1. Typical physical quantities of anemone jets in each layer of the solar atmosphere.

\begin{tabular}{cccccc}
\hline Region & Length $(\mathrm{km})$ & Velocity $\left(\mathrm{kms}^{-1}\right)$ & Lifetime $(\mathrm{s})$ & Alfvén velocity $\left(\mathrm{km} \mathrm{s}^{-1}\right)$ & $t / t_{A}$ \\
\hline Corona & $10^{4}-10^{5}$ & $100-10^{3}$ & $10^{3}-10^{4}$ & $100-10^{3}$ & $10-100$ \\
Chromosphere & $10^{3}-10^{4}$ & $10-100$ & $100-10^{3}$ & $10-100$ & $10-50$ \\
Photosphere? & $10-100$ & $1-10$ & $10-100$ & $1-10$ & $10 ?$ \\
\hline
\end{tabular}

\section{Method}

Although in the solar photosphere the temperature is $\sim 6000 \mathrm{~K}$, ionization degree is low, and majority of components of the fluid are neutral, numerous collisions do occur between the neutral and plasma owing to the high density in the photosphere. The collision time between the ion and neutral $\tau$ represents $\nu_{i n}^{-1} \simeq n_{n} \sqrt{ }\left(8 k_{B} T / \pi m_{i n}\right) \Sigma_{i n}$, where $\nu_{i n}, n_{n}, m_{i n}=\left(m_{i}+m_{n}\right) / m_{i} m_{n}$, and $\Sigma_{i n} \simeq 5 \times 10^{-15} \mathrm{~cm}^{2}$ are the collisional frequency of ions with the neutrals, number density of the neutrals, reduced mass of the ion and neutral, and ion-neutral collision cross-sections (Leake \& Arber 2006). We assume that all the elements are hydrogen and $n_{n}=10^{17} \mathrm{~cm}^{-3}$ in the photosphere; then, $m_{i n}$ is the proton mass $m_{p}$ and $\tau \simeq 10^{-9} \mathrm{~s}$. This time is considerably shorter than the time resolution of our simulation $d t \sim 0.1 \times 0.1 \mathrm{~km} /\left(c_{s}=8.15 \mathrm{~km} \mathrm{~s}^{-1}\right) \sim 10^{-3} \mathrm{~s}$. Thus, we can neglect the effect of the partially ionized plasma in our simulation, and we adopt the one fluid (MHD) approximation.

For our numerical simulation, we use Athena++ code with the van Leer predictorcorrector scheme and Piecewise Linear Method (Stone et al. 2020). We solve the ideal MHD equations including uniform gravity and simple form radiative cooling (Newton cooling). The basic equations are as follows.

$$
\begin{aligned}
& \frac{\partial \rho}{\partial t}+\boldsymbol{\nabla} \cdot(\rho \boldsymbol{v})=0 \\
& \frac{\partial \rho \boldsymbol{v}}{\partial t}+\boldsymbol{\nabla} \cdot(\rho \boldsymbol{v} \boldsymbol{v})=-\nabla p+\frac{1}{4 \pi}(\boldsymbol{\nabla} \times \boldsymbol{B}) \times \boldsymbol{B}+\rho \boldsymbol{g} \\
& \left.\frac{\partial\left(e+\frac{1}{2} \rho v^{2}+\frac{B^{2}}{8 \pi}\right)}{\partial t}+\boldsymbol{\nabla} \cdot\left[\left(h+\frac{1}{2} \rho v^{2}+\frac{B^{2}}{4 \pi}\right) \boldsymbol{v}-\frac{1}{4 \pi}(\boldsymbol{B} \cdot \boldsymbol{v}) \boldsymbol{B}\right)\right] \\
& =\rho \boldsymbol{g} \cdot \boldsymbol{v}-\frac{\rho R}{\mu(\gamma-1)} \frac{T-T_{0}}{\tau_{\text {cooling }}} \\
& \frac{\partial \boldsymbol{B}}{\partial t}=\boldsymbol{\nabla} \times(\boldsymbol{v} \times \boldsymbol{B}) \\
& p=\frac{\rho R T}{\mu}
\end{aligned}
$$

, where $e$ is the internal energy of the fluid, $h=p+e$ is the enthalpy, $T_{0}=6000 \mathrm{~K}$ is uniform initial temperature, $\tau_{\text {cooling }}=1 \mathrm{~s}$ is mean cooling time and $\mu=1.25$ is mean molecular weight in the solar photosphere. Note that $\tau_{\text {cooling }}=1 \mathrm{~s}$ agrees with the cooling time deduced for 
Table 2. Units used in numerical simulation.

\begin{tabular}{ccccccc}
\hline Length & Density & Temperature & Velocity & Gas pressure & Magnetic field & Time \\
\hline$L_{0}$ & $\rho_{0}$ & $T_{0}$ & $v_{0}$ & $p_{0}$ & $B_{0}$ & $t_{0}$ \\
$10 \mathrm{~km}$ & $10^{-7} \mathrm{~g} \mathrm{~cm}^{-3}$ & $6000 \mathrm{~K}$ & $6.32 \times 10^{5} \mathrm{~cm} \mathrm{~s}^{-1}$ & $3.97 \times 10^{4} \mathrm{erg} \mathrm{cm}^{-3}$ & $706 \mathrm{G}$ & $1.57 \mathrm{~s}$
\end{tabular}

the photosphere with temperature $T=6000 \mathrm{~K}$ (Athay 1976, p. 474 table X-4), and that it decreases with height in the actual sun. In our simulation, because magnetic reconnection occurs at a height of approximately $20 \mathrm{~km}$ and the majority of the temperature change occurs in this vicinity, we set the cooling time uniform within the simulation box for simplicity. $g$ is the gravitational acceleration of the solar atmosphere $\left(|\boldsymbol{g}|=2.7 \times 10^{4} \mathrm{~cm} \mathrm{~s}^{-2}\right)$ and we set $\boldsymbol{g}=\left(g_{x}, g_{y}, g_{z}\right)=(-g, 0,0)$. We assume a specific heat ratio of $\gamma=5 / 3$.

For the normalization of the simulation, we use the typical length $L_{0}=10 \mathrm{~km}$, initial temperature $T_{0}=6000 \mathrm{~K}$, and density in the bottom boundary $\rho_{0}=1.0 \times 10^{-7} \mathrm{~g} \mathrm{~cm}^{-3}$. Then, we use the unit of velocity, pressure, magnetic field, and time as $v_{0}=\sqrt{ }\left(R T_{0} / \mu\right)=6.32 \times$ $10^{5} \mathrm{cms}^{-1}, p_{0}=\rho_{0} v_{0}^{2}=3.97 \times 10^{4} \mathrm{ergcm}^{-3}, B_{0}=\sqrt{ }\left(4 \pi p_{0}\right)=706 \mathrm{G}$, and $t_{0}=L_{0} / v_{0}=1.57 \mathrm{~s}$ (see table $2)$. Note that $v_{0}$ is different from the sound speed in the solar photosphere $c_{s 0}=\sqrt{ }\left(\gamma R T_{0} / \mu\right)=$ $8.15 \times 10^{5} \mathrm{cms}^{-1}$.

As an initial condition, we assume hydrostatic equilibrium with uniform temperature $T_{0}$. Moreover, we present a potential field as follows (Wyper et al. 2018).

$$
\begin{aligned}
& \boldsymbol{B}=\left(c_{1} \cos \theta, c_{1} \sin \theta, 0\right)+\sum_{i=1,16} \boldsymbol{\nabla} \times \boldsymbol{A}_{i} \\
& \boldsymbol{A}_{i}=\frac{b_{i} x_{i}^{3}}{2\left[x_{i}^{\prime 2}+\left(y_{i}^{\prime}-y_{c}\right)^{2}+z_{i}^{\prime 2}\right]^{3 / 2}} \times\left[-z_{i}^{\prime} \boldsymbol{e}_{y}+\left(y_{i}^{\prime}-y_{c}\right) \boldsymbol{e}_{z}\right]
\end{aligned}
$$

$c_{1}=-0.8$ and $\theta=-22^{\circ}$ are the parameters of the background field strength and angle, respectively. $x_{i}^{\prime}=x-x_{i}, y_{i}^{\prime}=y-y_{i}, z_{i}^{\prime}=z-z_{i}$. We present the values of $b_{i}, x_{i}, y_{i}, z_{i}$ in table3. Figure 1 (a) displays this initial field.

We set the velocity field in the bottom boundary as follows (Pariat et al. 2009; Wyper et al. 2018):

$$
\begin{aligned}
v_{x} & =0 \\
\boldsymbol{v}_{\perp} & =f(t) \nu_{0} g\left(B_{x}\right) \boldsymbol{e}_{x} \times \nabla B_{x} \\
g\left(B_{x}\right) & =k \frac{B_{r}-B_{l}}{B_{x}} \tanh \left(k \frac{B_{x}-B_{l}}{B_{r}-B_{l}}\right) \quad B_{l} \leq B_{x} \leq B_{r} \\
& =0 \quad \text { otherwise. }
\end{aligned}
$$

We set $k=4.0, \nu=0.002, B_{l}=0.15$, and $B_{r}=3.6 . f(t)$ is the time development of the bottom boundary photospheric motion, and we assume the following: 

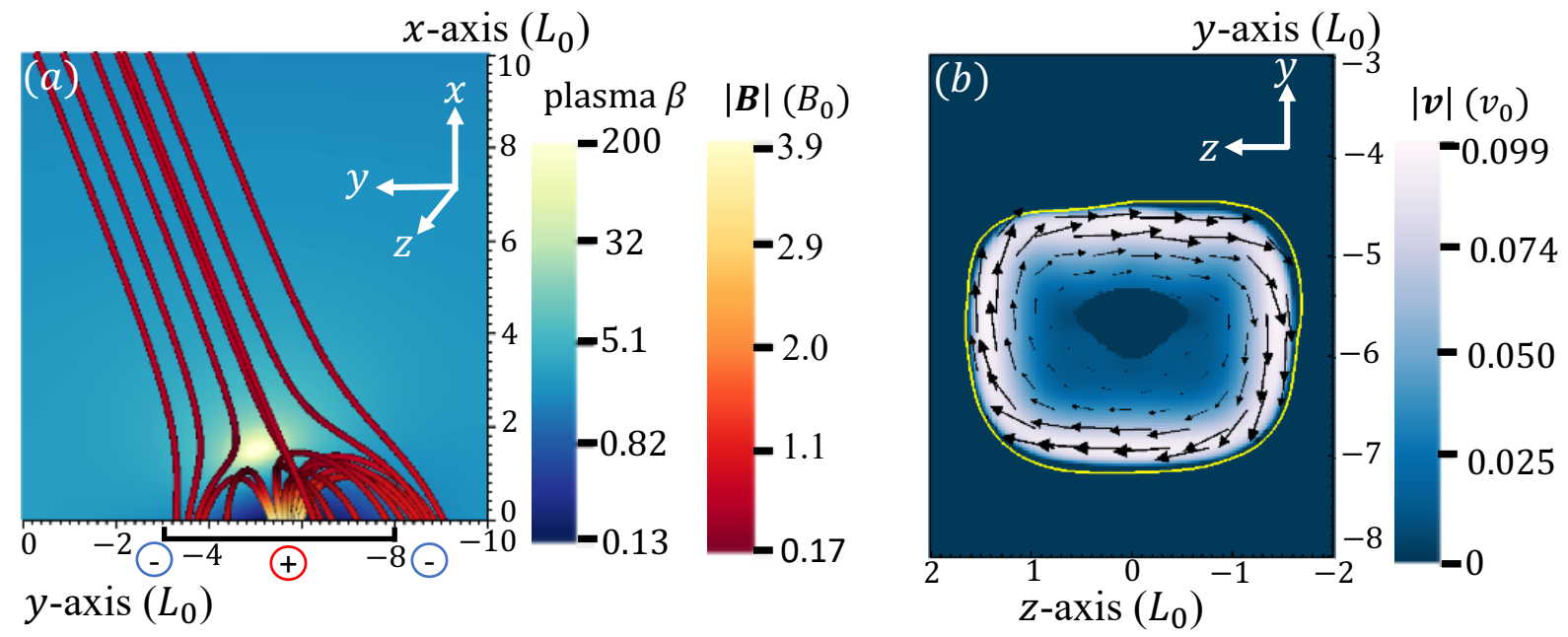

Fig. 1. (a): Initial potential field and distribution of plasma $\beta$. The symbols in the bottom boundary mean the polarity of the magnetic field. The region indicated by the black line along the $y$-axis represents the range of the (b). (b): Velocity distribution in the bottom boundary at $t=60 t_{0}$. Yellow contour means polarity inversion line.

Table 3. Parameters of magnetic field.

\begin{tabular}{|c|c|c|c|c|}
\hline$i$ & $b_{i}$ & $x_{i}$ & $y_{i}$ & $z_{i}$ \\
\hline 1 & -1.35 & -1.0 & -0.5 & -1.0 \\
\hline 2 & -1.35 & -1.0 & -0.5 & -0.5 \\
\hline 3 & -1.35 & -1.0 & -0.5 & 0 \\
\hline 4 & -1.35 & -1.0 & -0.5 & 0.5 \\
\hline 5 & -1.35 & -1.0 & -0.5 & 1.0 \\
\hline 6 & -1.35 & -1.0 & 0 & -1.0 \\
\hline 7 & -1.35 & -1.0 & 0 & 0 \\
\hline 8 & -1.35 & -1.0 & 0 & 1.0 \\
\hline 9 & -1.17 & -1.0 & -1.5 & -1.0 \\
\hline 10 & -1.17 & -1.0 & -1.5 & -0.5 \\
\hline 11 & -1.17 & -1.0 & -1.5 & 0 \\
\hline 12 & -1.17 & -1.0 & -1.5 & 0.5 \\
\hline 13 & -1.17 & -1.0 & -1.5 & 1.0 \\
\hline 14 & -1.17 & -1.0 & -1.0 & -1.0 \\
\hline 15 & -1.17 & -1.0 & -1.0 & 0 \\
\hline 16 & -1.17 & -1.0 & -1.0 & 1.0 \\
\hline
\end{tabular}

* Meaning of $b_{i}, x_{i}, y_{i}$, and $z_{i}$ is provided in the text. 


$$
\begin{aligned}
f(t) & =t / t_{1} \quad\left(t<t_{1}\right) \\
& =1 \quad\left(t_{1} \leq t<t_{2}\right) \\
& =1-\frac{t-t_{2}}{t_{1}} \quad\left(t \leq t_{2}\right)
\end{aligned}
$$

, where $t_{1}=60 t_{0}$ and $t_{2}=75 t_{0}$. Note that although the adopted function is the same, the values of these parameters are different from those of the previous study, resulting in different values of the velocity field from those of the previous study. These values are based on the assumption that granulation satisfies the Kolmogorov law $\epsilon \sim v_{\lambda}^{3} / \lambda$. Specifically, the typical size of the granulation is $1000 \mathrm{~km}$ and the speed is $1 \mathrm{~km} \mathrm{~s}^{-1}$; then, the speed of the smaller vortex of approximately $10 \mathrm{~km}$ is determined from the Kolgomogorov law as follows:

$$
v_{\lambda=10 \mathrm{~km}} \sim v_{\lambda=1000 \mathrm{~km}}\left(\frac{\lambda=10 \mathrm{~km}}{\lambda=1000 \mathrm{~km}}\right)^{1 / 3} \sim 0.25 \mathrm{kms}^{-1} .
$$

Moreover, the time scale of the $10 \mathrm{~km}$ scale small vortex is

$$
t_{\lambda=10 \mathrm{~km}} \sim \frac{\lambda=10 \mathrm{~km}}{v_{\lambda=10 \mathrm{~km}}} \sim 40 \mathrm{~s} .
$$

These values are consistent with the velocity and lifetime used in our simulation within a factor three. Figure 1 (b) displays this boundary motion. For gas pressure and density, the initial values are maintained, and for the magnetic field, the gradient is set to zero. In the other boundary, we use an open boundary and set the gradient of the physical value as zero.

We set our simulation box as $\left[x_{\min }, x_{\max }\right] \times\left[y_{\min }, y_{\max }\right] \times\left[z_{\min }, z_{\max }\right]=\left[0,50 L_{0}\right] \times$ $\left[-12.5 L_{0}, 7.5 L_{0}\right] \times\left[-5 L_{0}, 5 L_{0}\right]$. We set the grid size as $d x=d y=d z=0.05$. Moreover, the lattice spacing is halved at $\left[0,10 L_{0}\right] \times\left[-12.5 L_{0},-2.5 L_{0}\right] \times\left[-2.5 L_{0}, 2.5 L_{0}\right]$ to sufficiently resolve the anemone-type magnetic field.

\section{Results}

Figure 2 displays the time evolution of the density distribution on the $z=0$ plane and magnetic field lines integrated in the plane. First, the initial magnetic fields are twisted to form a sheared loop because of the photospheric motion based on the boundary condition (figure 2a). As the magnetic pressure increases owing to the magnetic energy injected by the photospheric motion, the sheared loop rises (figure $2 \mathrm{~b}$ ). At this time, magnetic reconnection occurs at the top of the sheared loop, and a jet-like structure, which is an elongated density increase along the magnetic field lines and appears as a jet in imaging observations, is generated. This reconnection removes the magnetic field that holds the sheared loop from above and thus further promotes the rising of the sheared loop. When the twisted sheared loop emerges sufficiently, the twisted sheared 


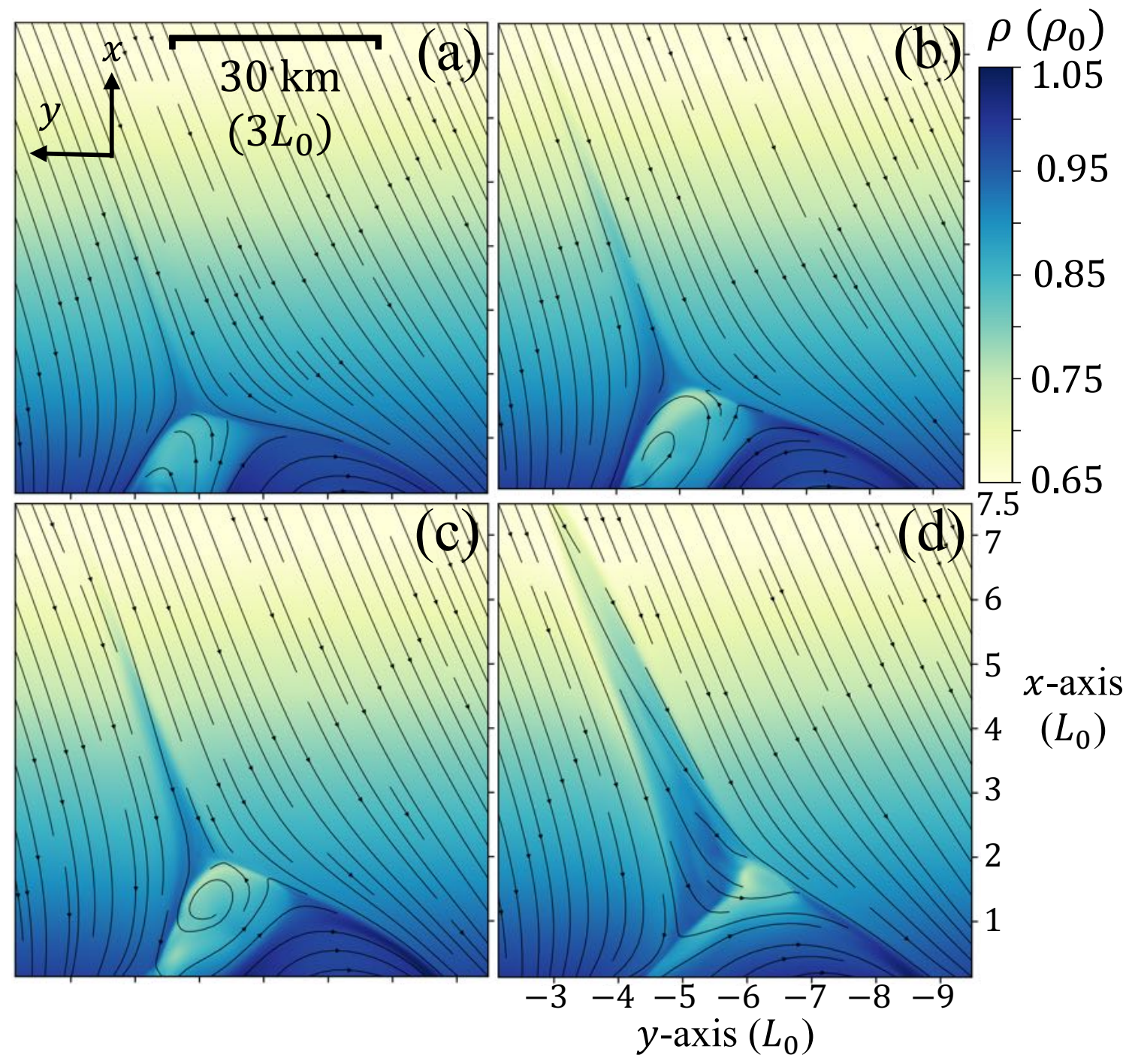

Fig. 2. Time development of density distribution in $z=0$ plane. (a): $t=60 t_{0}$, (b): $t=70 t_{0}$, (c): $t=80 t_{0}$, (d): $t=100 t_{0}$. Black lines display magnetic field lines integrated by $x, y$ components in the plane. The upper boundary is the $x=7.5 L_{0}$ plane, where we measure the MHD wave energy fluxes in figures 14 and 17. (An animation of this figure is available.) 
loop and the background field cause magnetic reconnection, resulting in an untwisting jet-like structure, which releases numerous Alfvén waves (figures 2c and 2d). This mechanism is similar to coronal jet simulation (e.g., Pariat et al. 2009; Archontis \& Hood 2013; Wyper et al. 2018), which assumes a corona of $\beta \ll 1$.

The length, apparent speed, and lifetime of this jet-like structure are approximately $100 \mathrm{~km}, 5 \mathrm{~km} \mathrm{~s}^{-1}$, and $80 \mathrm{~s}$. This apparent speed approximately corresponds to Alfvén speed at this point. The length of the twisted sheared loop is approximately $30 \mathrm{~km}$, and hence the Alfvén time $t_{A}$ is $t_{A} \sim 30 \mathrm{~km} / 5 \mathrm{kms}^{-1} \sim 6 \mathrm{~s}$. Thus, the lifetime $t$ divided by the Alfvén time $t_{A}$ is $t / t_{A} \sim 80 \mathrm{~s} / 6 \mathrm{~s} \sim 13.3$. All of these values are consistent with those in table 1 .

Figure 3 indicates how much the density and temperature increase in the jet-like structure. Figures $3 \mathrm{a}$ and $3 \mathrm{~b}$ indicate that the jet-like structure has a density of approximately 1.1 times greater than its surroundings. Furthermore, figures $3 \mathrm{c}$ and $3 \mathrm{~d}$ indicate that the temperature increases approximately $200 \mathrm{~K}$ in the jet-like structure, and that the temperature increase has an inverted-Y shape.

Figure 4 displays the 3D appearance at $t=81 t_{0}$. From figure $4 \mathrm{~b}$, we can observe that magnetic reconnection occurs between the twisted sheared loop and background fields, and plasma frozen in the reconnected field lines is accelerated approximately in the z-direction; that is, in the direction normal to the ambient field. Moreover, the twisted loop, background field, and current sheet are aligned in the z-direction. Therefore, the reconnection process can be investigated in more detail with an appropriate $y=$ const cross section, though it is actually a complex 3D phenomenon.

Figures 5 and 6 display the physical values of the jet-like structure in the $y=-4.95 L_{0}$ plane, where we can interpret the process of magnetic reconnection in a broadly $2 \mathrm{D}$ view. Closed fields at the bottom indicate the twisted sheared loop. From figure 5a, we can observe reconnection inflow and outflow. The outflow's velocity is $\sim 0.7 v_{0}$, which corresponds to Alfvén velocity in the sheared loop (figure $5 \mathrm{~b}$ ). The inflow's velocity is $\sim 0.1 v_{0}$. Then, we can estimate the reconnection rate $v_{\text {in }} / v_{\text {out }} \sim 0.1$. From figure $5 \mathrm{c}$, we can observe that the maximum temperature in the current sheet is $\sim 6600 \mathrm{~K}$ and the outflow temperature is $\sim 6300 \mathrm{~K}$. From figure $5 \mathrm{~d}$, we can observe the area of $\operatorname{div} v<0$ between the inflow and outflow, which corresponds to the compression occurring. Moreover, the gas pressure becomes stronger and the magnetic field becomes weaker before and after the $\operatorname{div} v<0$ region (figures $6 \mathrm{~b}$ and $6 \mathrm{c}$ ), which indicates that the $\operatorname{div} v<0$ corresponds to a slow shock formed by magnetic reconnection. The slow shock and the reconnection rate in our simulation imply that this reconnection has similar properties to those of the Petschek model (Petschek 1964). 


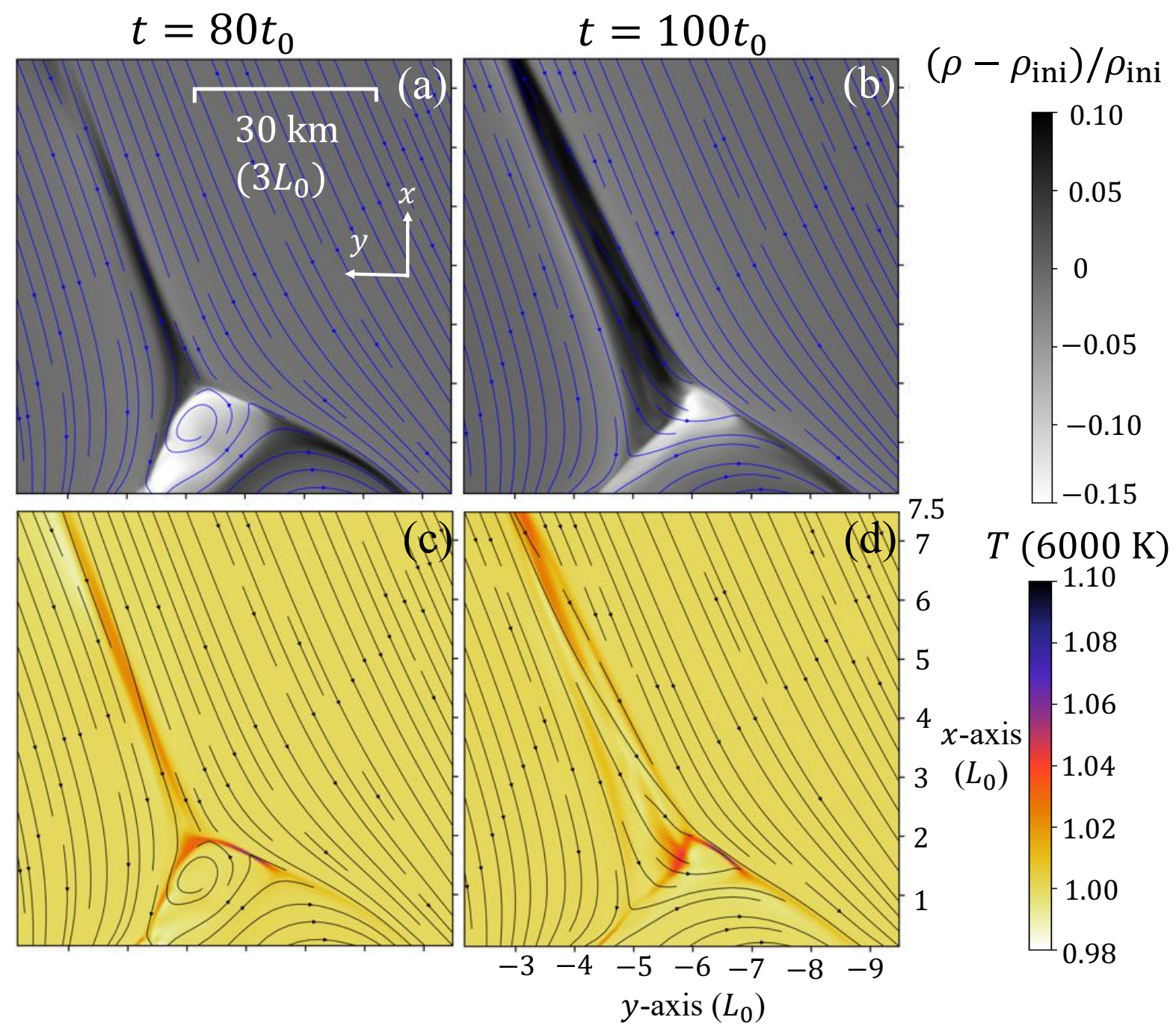

Fig. 3. Rate of increase in density and temperature of the jet-like structure in $z=0$ plane. The left and right columns indicate the same time as in figures $2 \mathrm{c}$ and $2 \mathrm{~d}$, respectively. $\rho_{\mathrm{ini}}$ is the initial density. The upper boundary is the $x=7.5 L_{0}$ plane, where we measure the MHD wave energy fluxes in figures 14 and 17.

From figures $5 \mathrm{e}-\mathrm{h}$, we can observe that the slow shock is displaced upward and the plasma is accelerated approximately in the z-direction in the upper atmosphere. This can certainly be confirmed by the one-dimensional (1D) distribution displayed in figure 7, which indicates both the upward and downward slow shocks formed by reconnection. Note that this displacement of the slow shock is not a result of the upward propagation of the slow shock in this plane. Figure 8 presents the time development of the $\operatorname{div} v<0$ region and gas pressure in the $z=0$ and $y=-4.95 L_{0}$ planes. From this figure, we can observe that the region of $\operatorname{div} v<0$ exists along the direction of the jet-like structure, that is, a uniform background magnetic field. Moreover, the strong $\operatorname{div} v<0$ region extends from near the region where magnetic reconnection is occurring. These facts indicate that the $\operatorname{div} v<0$ region is formed by the propagation of the 

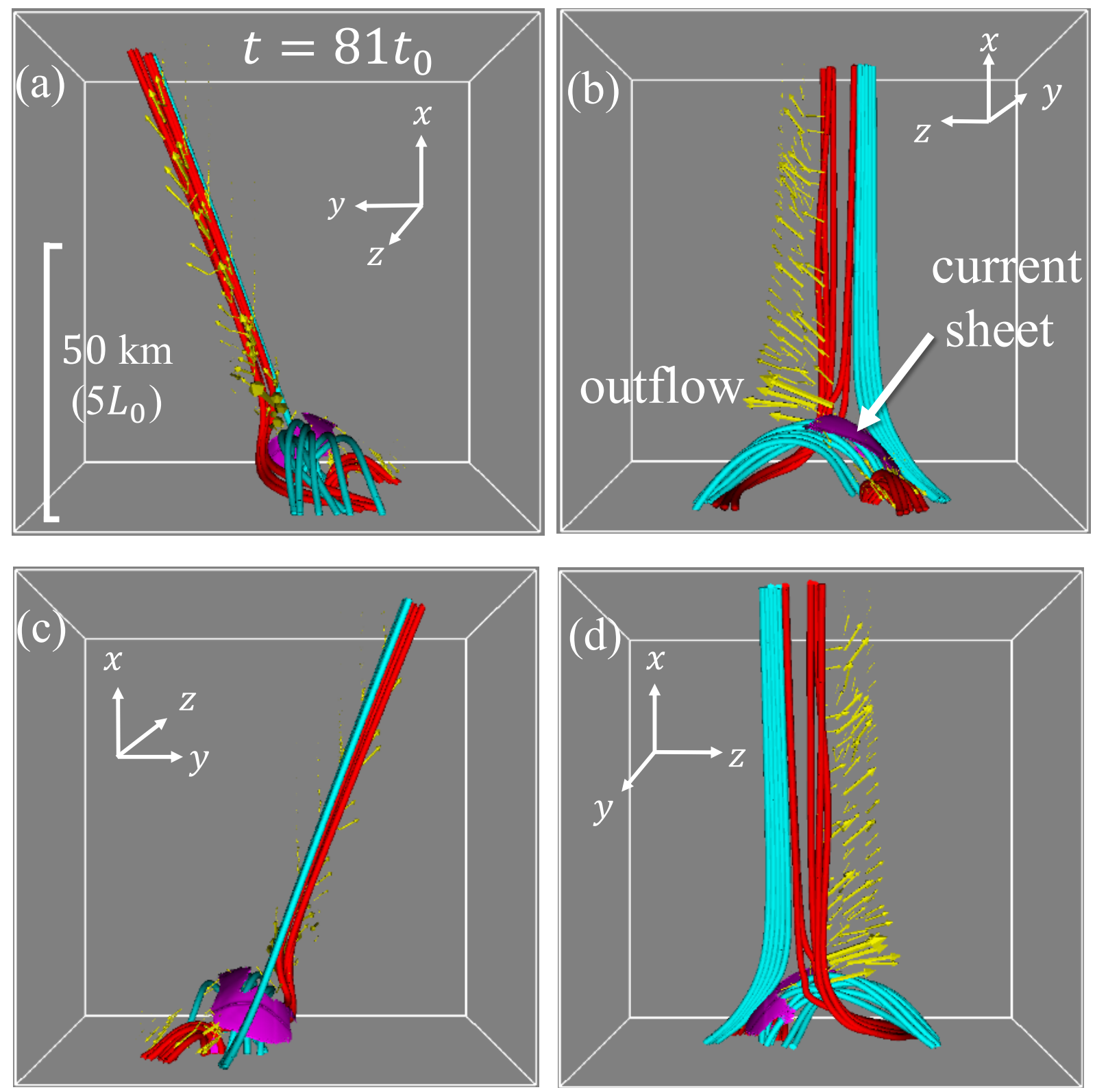

Fig. 4. 3D view of the jet-like structure in $t=81 t_{0}$. Yellow arrows and pink surface are the velocity and current sheet. Light blue lines indicate twisted sheared loop and open fields before magnetic reconnection. Red lines indicate reconnected open fields and post-flare loop. The size of the largest arrow corresponds to $0.6 v_{0}$. 

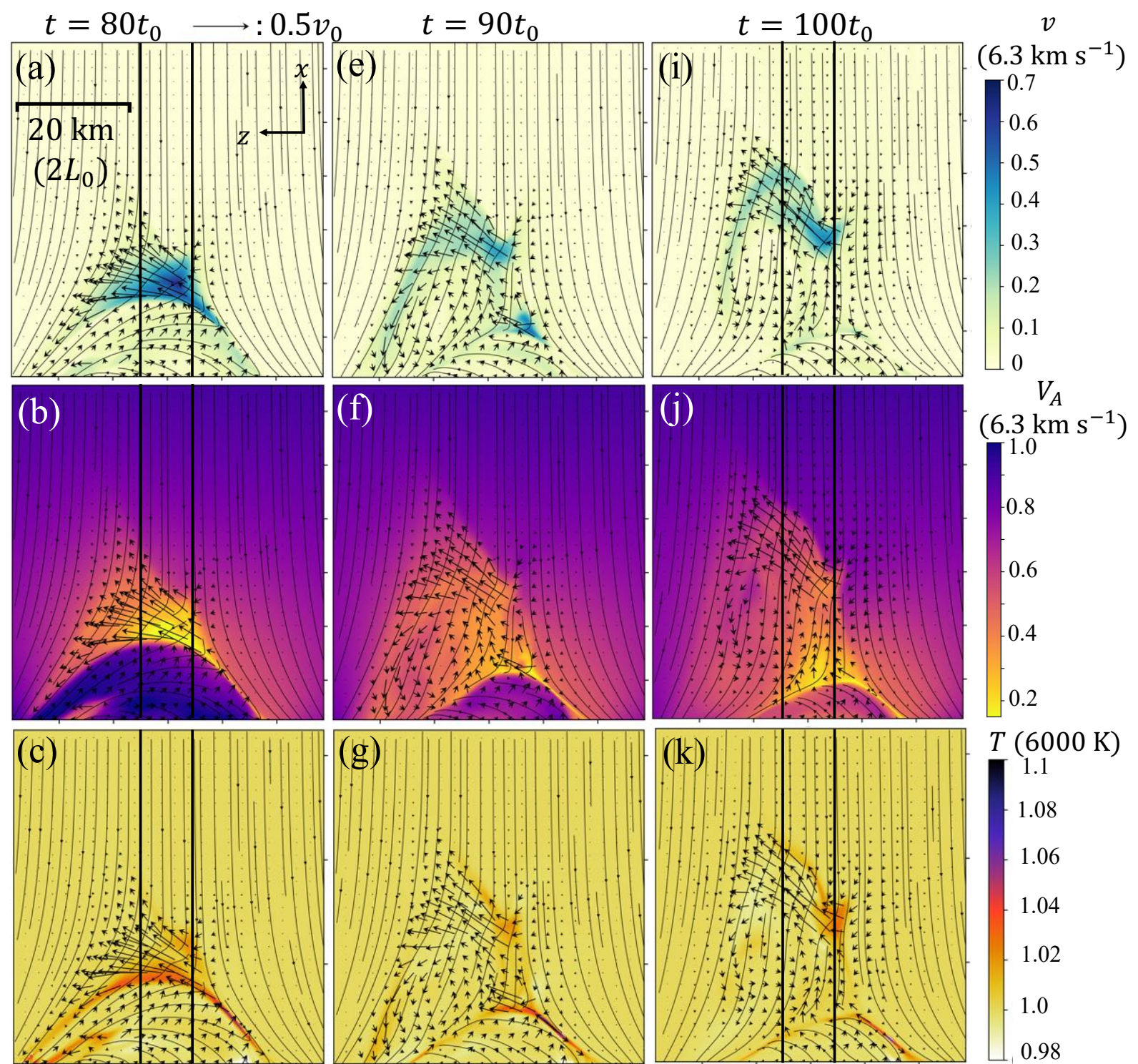

$T(6000 \mathrm{~K})$
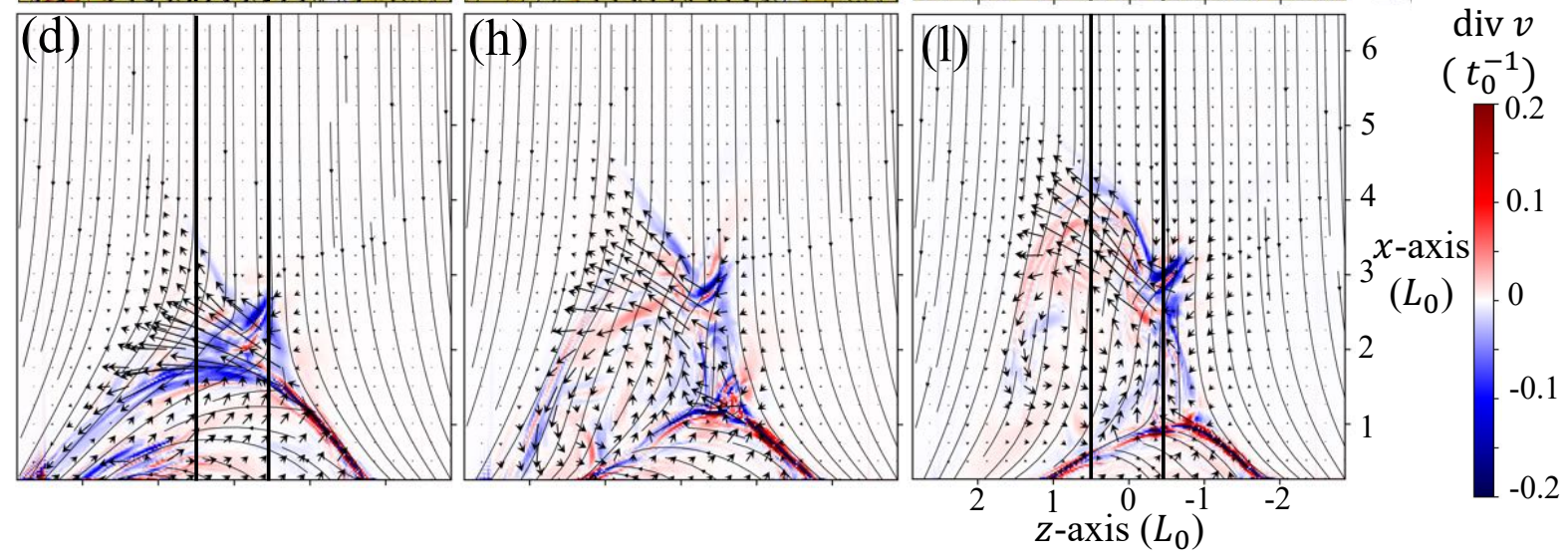

Fig. 5. Physical values in $y=-4.985 L_{0}$ plane at $t=80 t_{0}((\mathrm{a})-(\mathrm{d})), t=90 t_{0}((\mathrm{e})-(\mathrm{h}))$, and $t=100 t_{0}((\mathrm{i})-(\mathrm{l}))$. Each row shows velocity ((a), (e), and (i)), Alfvén velocity ((b), (f), and (j)), temperature ((c), (g), and (k)) and divergence of velocity $((\mathrm{d}),(\mathrm{h})$, and $(\mathrm{l}))$. Black arrows are velocity in the plane. Black lines show magnetic field lines integrated by $x, z$ components in the plane. One dimensional distributions of some physical quantities along two black solid lines in $t=80 t_{0}$ and $100 t_{0}$ are shown in figure 7. (An animation of (d), (h), and (I) is available.) 

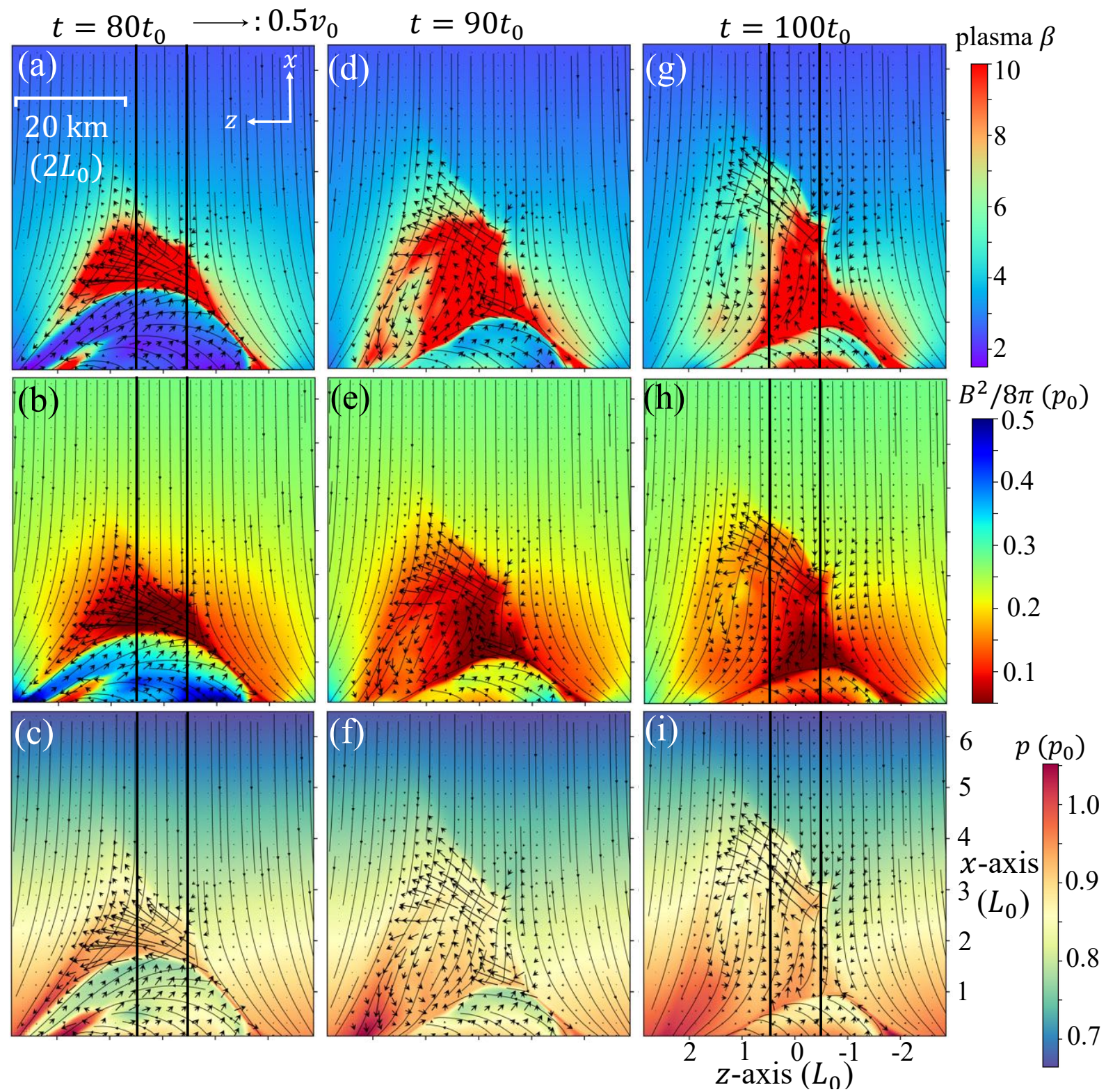

Fig. 6. Physical values in $y=-4.985 L_{0}$ plane at $t=80 t_{0}((\mathrm{a})-(\mathrm{c})), t=90 t_{0}((\mathrm{~d})-(\mathrm{f}))$, and $t=100 t_{0}((\mathrm{~g})-(\mathrm{i}))$. Each row shows plasma $\beta((\mathrm{a})$, (d), and (g)), magnetic pressure ((b), (e), and (h)), and gas pressure ((c), (f), and (i)). Black arrows are velocity in the plane. Black lines show magnetic field lines integrated by $x, z$ components in the plane. One dimensional distributions of some physical quantities along two black solid lines in $t=80 t_{0}$ and $100 t_{0}$ are shown in figure 7. 


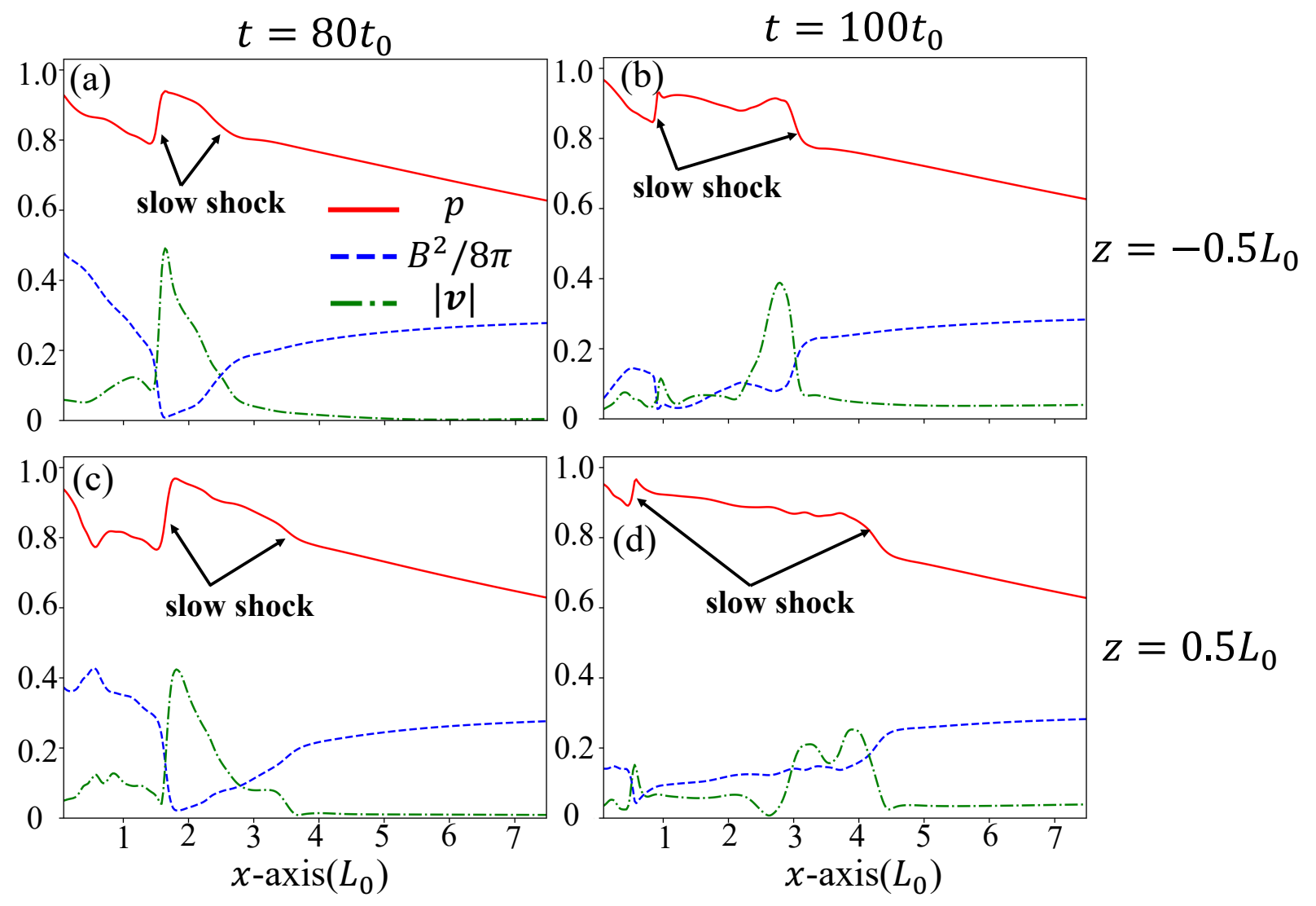

Fig. 7. One-dimensional (1D) distribution of gas pressure, magnetic pressure, and velocity along the solid black lines at $z=0.5$ and -0.5 shown in figures 5 and 6 . The solid red lines indicate gas pressure, the dashed blue lines indicate magnetic pressure, and the dash-dot green lines indicate speed. The left column shows the distributions at $t=80$, and the right column shows the distributions at $t=100$. (a) and (b) correspond to distributions at $z=-0.5$, and (c) and (d) correspond to distributions at $z=0.5$. The units are $3.97 \times 10^{4} \mathrm{erg} \mathrm{cm}^{-3}$ for gas and magnetic pressure and $6.32 \times 10^{5} \mathrm{~cm} \mathrm{~s}^{-1}$ for plasma speed.

slow shock produced in the process of magnetic reconnection along a uniform background field. Furthermore, from the figures $8 \mathrm{a}-\mathrm{c}$, we can observe that the sheared loop is displaced in the negative y-axis direction with time, and with it, the region of $\operatorname{div} v<0$ is also displaced in the negative y-axis direction. It can be understood that the upward displacement of the slow shock in the $y=$ const cross section observed in figures 5e-h corresponds to the negative y-axis displacement of the slow shock propagating through a uniform background magnetic field. In fact, the slow shock rising speed in figure 5 is less than $0.1 v_{0}$, which corresponds to the sheared loop speed, namely the reconnection inflow. 

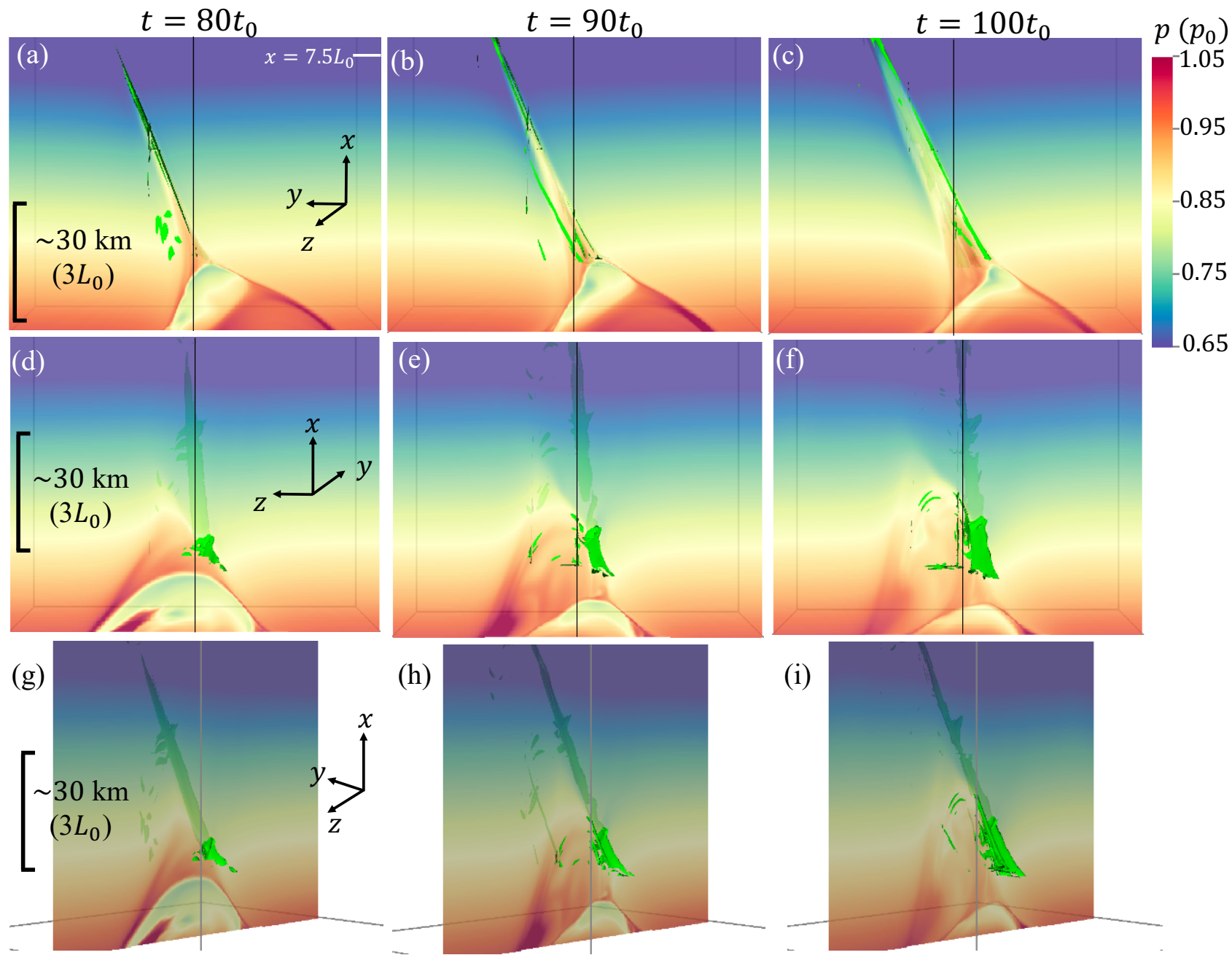

(h)

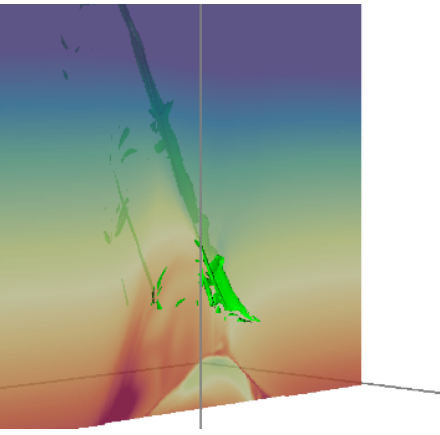

(i)
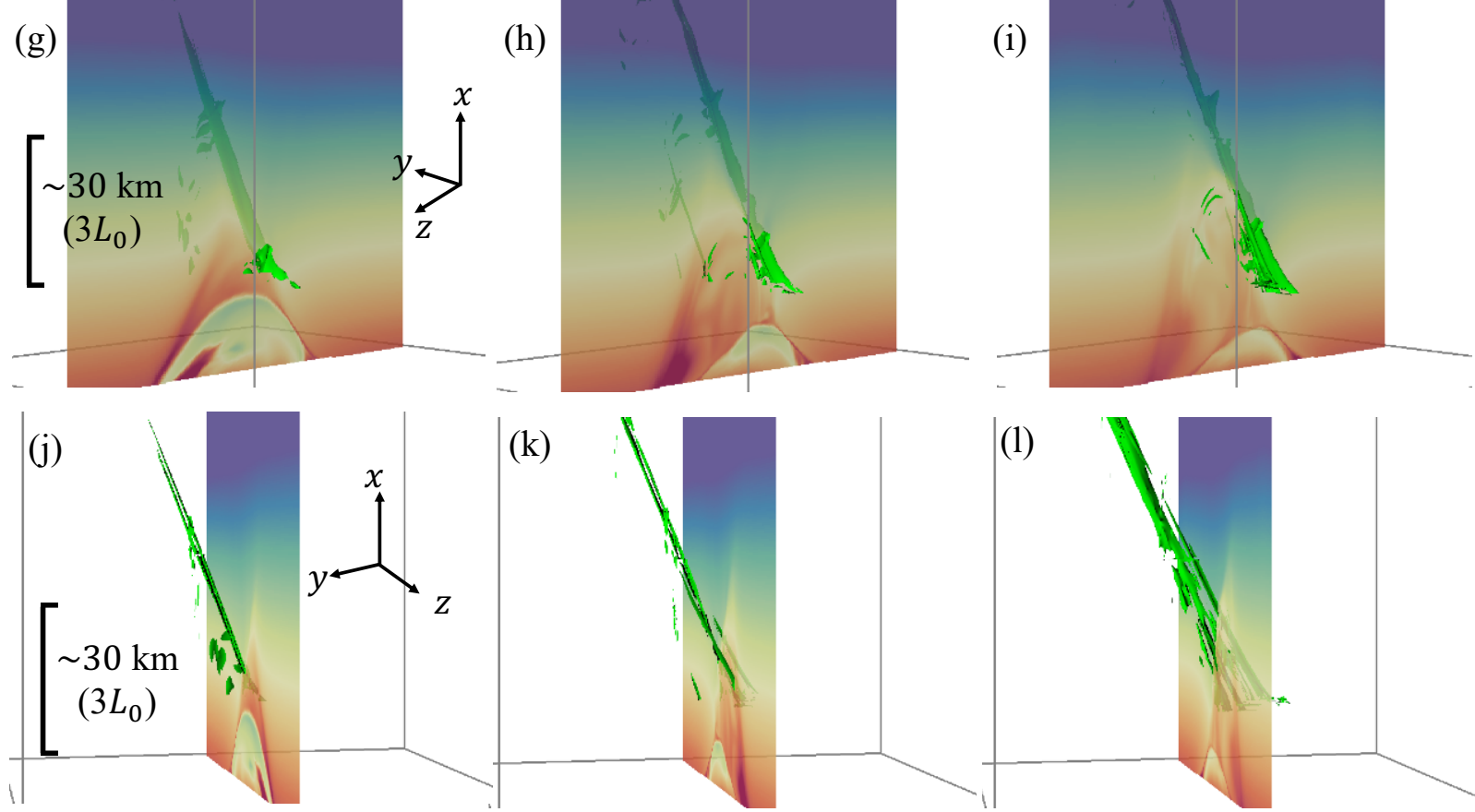

$(\mathrm{k})$
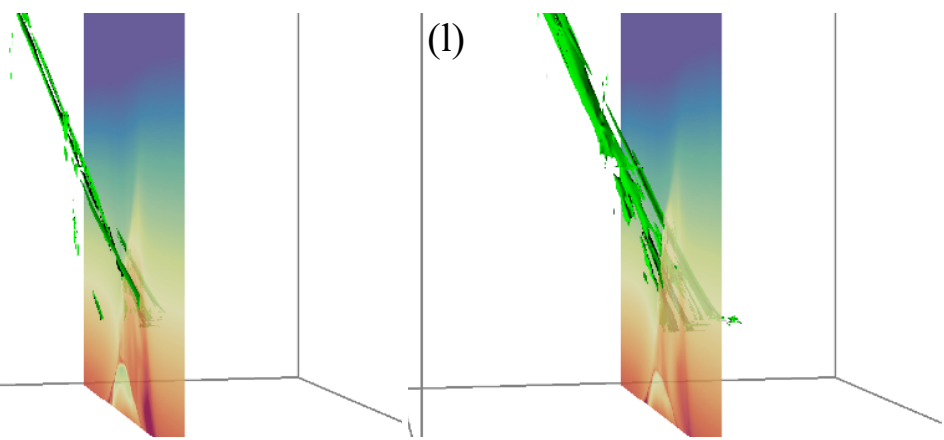

Fig. 8. Time development of the $\operatorname{div} v<0$ region and the gas pressure distribution shown in $2 \mathrm{D}$ planes. In each figure, the green surface indicates the regions of $\operatorname{div} v=-0.09 t_{0}^{-1}$ and $-0.07 t_{0}^{-1}$. (a)-(c): Gas pressure in the $z=0$ plane. The solid black line shown in the center of each figure shows the $y=-4.95 L_{0}$ plane. (d)-(f): Gas pressure in the $y=-4.95 L_{0}$ plane. The solid black line shown in the center of each figure shows the $z=0 L_{0}$ plane. (g)-(i): Gas pressure in the $y=-4.95 L_{0}$ plane and the 3D isosurfaces of $\operatorname{div} v$ from an oblique view. 

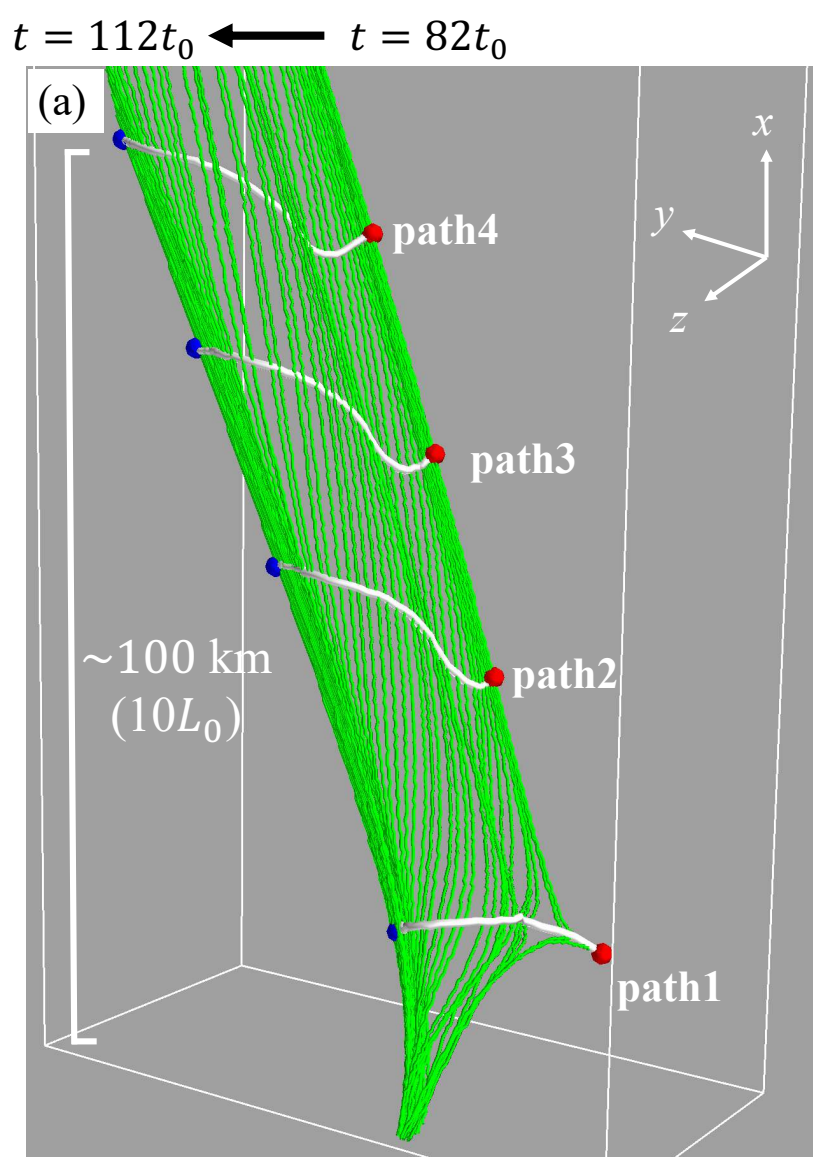

position $(10 \mathrm{~km})$
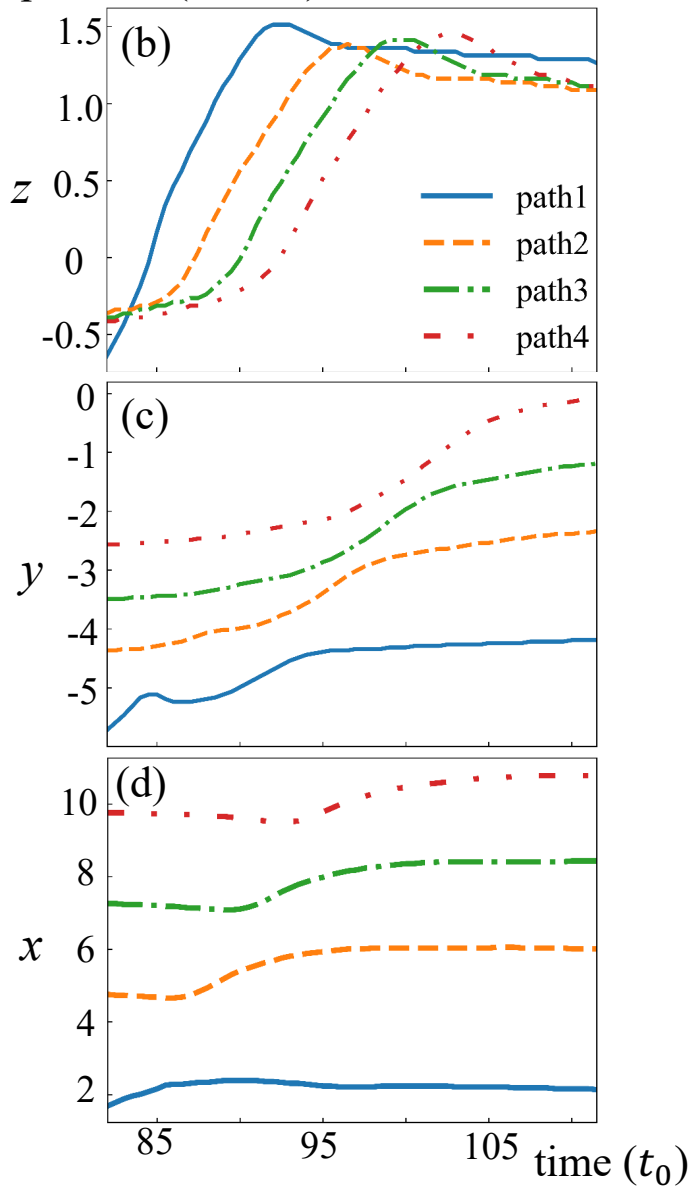

Fig. 9. (a): 3D view of the paths traced by Lagrangian methods. Red and blue points show the start and endpoints of time development. White lines mean each path and green lines show magnetic field lines passing through path 2 at each time. (b)-(d): Time development of $z$, $y$, and $x$ components of each path. The same type of lines indicates the same paths.

\section{Discussion}

\subsection{Formation mechanism of the jet-like structure}

To determine the formation mechanism of the jet-like structure, we display in figure 9a, the Lagrangian trajectories of typical fluid particles on the same reconnected magnetic field line. For determining a reconnected field line, we select a point where the total force in the $x$-direction is greater than $0.1 \rho_{0} v_{0}^{2} L_{0}^{-1}$ at $t=82 t_{0}$. Then, we select four points on the magnetic line passing through the point. We assume the time interval $d t$ is $0.5 t_{0}$. In figures $9 \mathrm{~b}-\mathrm{d}$, we indicate the position $(x, y, z)$ of those fluid particles on these as a function of time. From these figures, we can observe that the plasma rises; however, they do not move significantly when the magnetic tension attempts to straighten the reconnected field lines. In each path, the plasma particle is displaced only approximately $10 \mathrm{~km}$. This indicates that the jet-like structure of approximately 

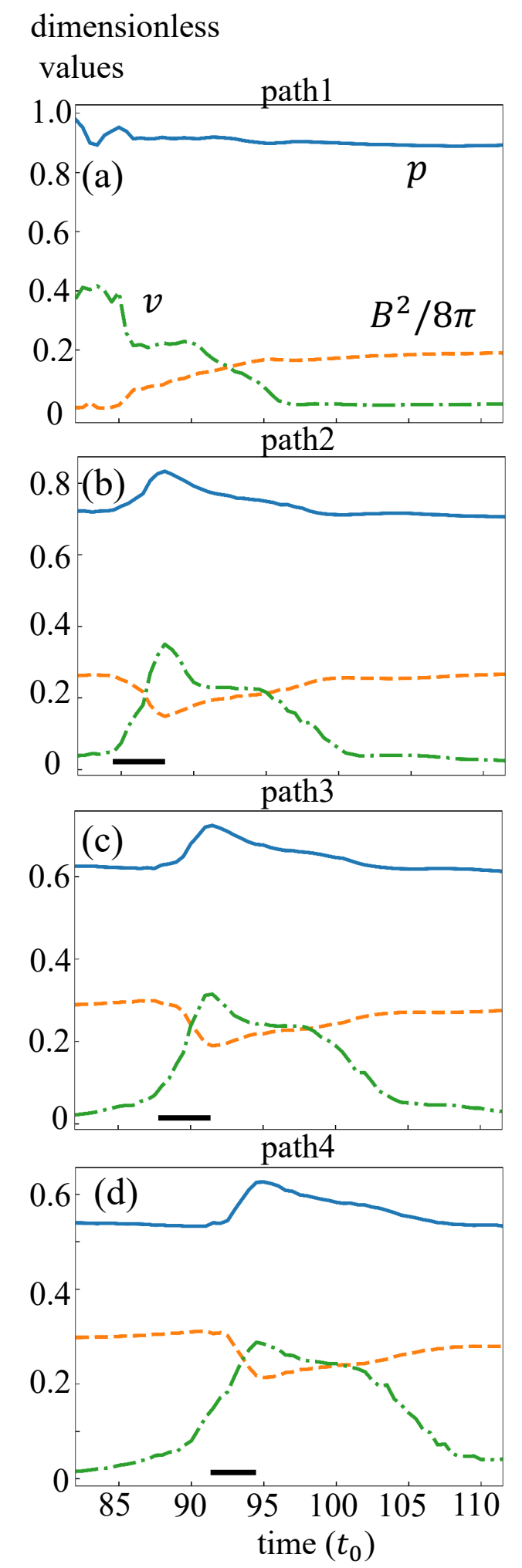

Fig. 10. Time development of physical values in each path tracked by the Lagrangian method. Solid blue, dashed orange, and dash-dot green lines mean gas pressure $p$, magnetic pressure $B^{2} / 8 \pi$, and plasma speed $|\boldsymbol{v}|$. The units are $3.97 \times 10^{4} \mathrm{erg} \mathrm{cm}^{-3}$ for gas and magnetic pressure and $6.32 \times 10^{5} \mathrm{~cm} \mathrm{~s}^{-1}$ for plasma speed. The black lines shown in (b), (c), and (d) indicate the time when the gas pressure increases in each path. 

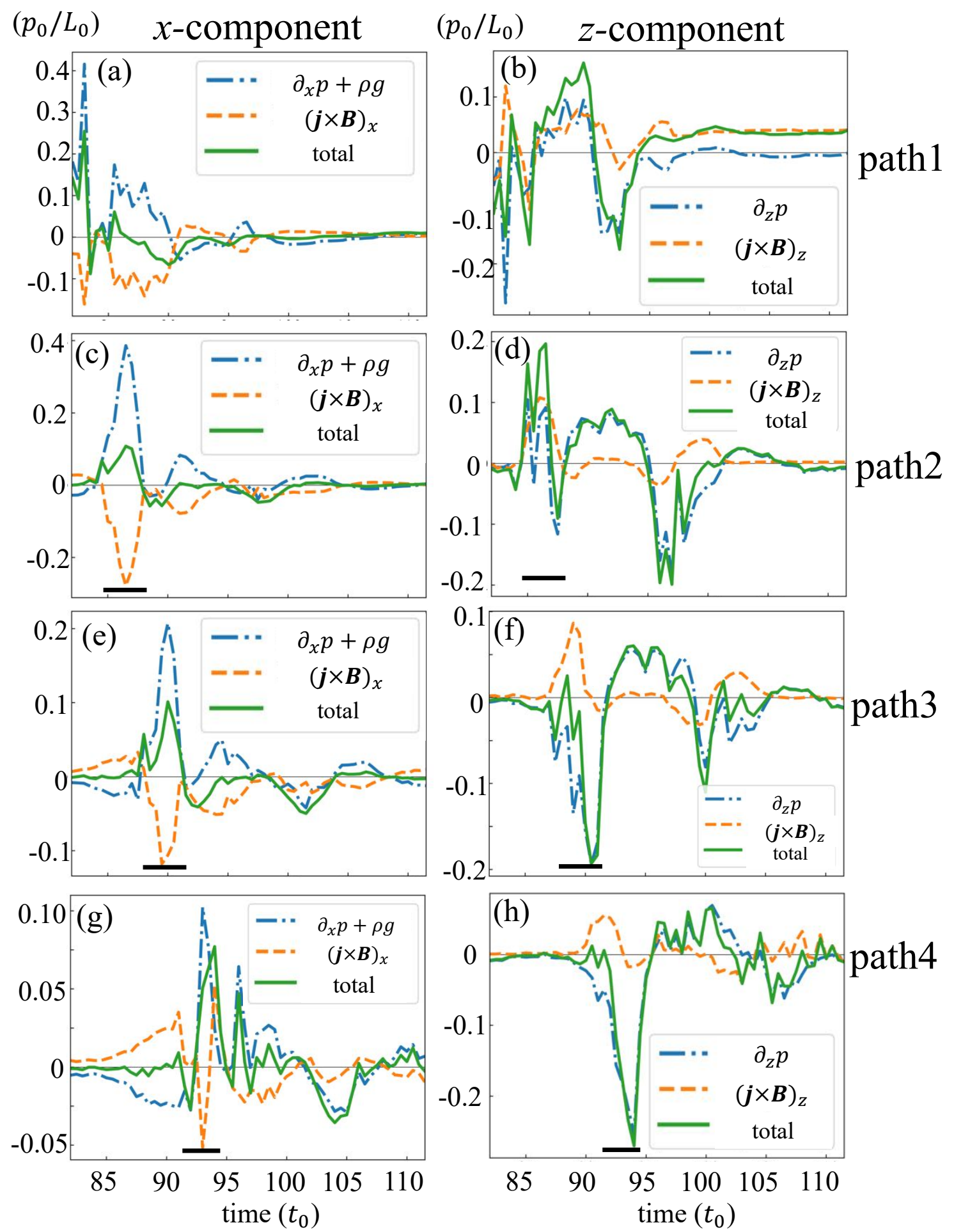

Fig. 11. Time evolution of the forces acting on the plasma on each path. The left column shows the $x$-component of the force, and the right column shows the $z$-component. Each row shows the respective path shown in Figure 9. Dash-dot blue, dashed orange, and solid green lines mean gas pressure gradient and gravity, Lorentz force, and total force. The black lines shown in (c)-(h) indicate the time when the gas pressure increases in each path in figure 10. 
$100 \mathrm{~km}$ observable in figures 2 and 3 is not formed by plasma motion; rather, it is formed by some wave propagation.

Figure 10 displays the time development of the gas pressure, magnetic pressure, and plasma speed along the trajectories indicated in figure 9. In figures 10b, 10c, and 10d, we can observe that gas pressure increases and the magnetic pressure decreases when the plasma speed increases rapidly. Furthermore, we can observe that this is the only time when the gas pressure increases in the time evolution of each path. From these facts, it can be understood that the gas compression is caused by the slow shock and the jet-like structure is the result of the slow shock propagating along a uniform background magnetic field observable in figures 5d, 5h, 5l, 8 , and 12 .

Figure 11 displays the temporal evolution of the forces acting on each path indicated in figure 9. We can observe that the upward gas pressure gradient is predominant in the $x$ component of the force when the gas pressure is increased. Conversely, in the $z$-component, the positive Lorentz force functions mainly at first; however, the difference with the gas pressure gradient is small, and the negative gas pressure gradient ultimately prevails. Considering that the magnetic field and gas pressure change rapidly in the slow shock and the value of plasma $\beta$ is approximately one, the behavior of these forces is consistent with the behavior when passing through the slow shock. After passing through the slow shock, the force acting on the plasma becomes increasingly less and settles into a new equilibrium state. These facts also indicate that the jet-like structure is not formed by reconnection outflow re-accelerated in the direction of the background magnetic field; rather, it is formed by compression due to the slow shock propagating in the background field's direction. That is, this jet-like structure is not mass motion; rather, it is a slow shock propagation.

Note that this formation mechanism is different from previous studies regarding coronal and chromospheric cases. In the coronal 2D simulation by Yokoyama \& Shibata (1996), hot jets are created by gas pressure increasing with a fast shock formed when reconnection outflow collides with the background field. In the 2D simulation in both the corona and chromosphere (Yokoyama \& Shibata 1996; Takasao et al. 2013), the magnetic centrifugal force working in reconnected field lines drives cool jets. In these cases, a slow shock propagates approximately in the direction of the reconnection outflow, and the outflow is re-accelerated in the direction of the background field by the gas pressure gradient or Lorentz force. These mechanisms are different from our photospheric case, where the slow shock propagates approximately along the reconnected field lines and the reconnection outflow is not re-accelerated. 3D simulation in the corona (e.g., Pariat et al. 2009) shows that jets are accelerated by nonlinear torsional 

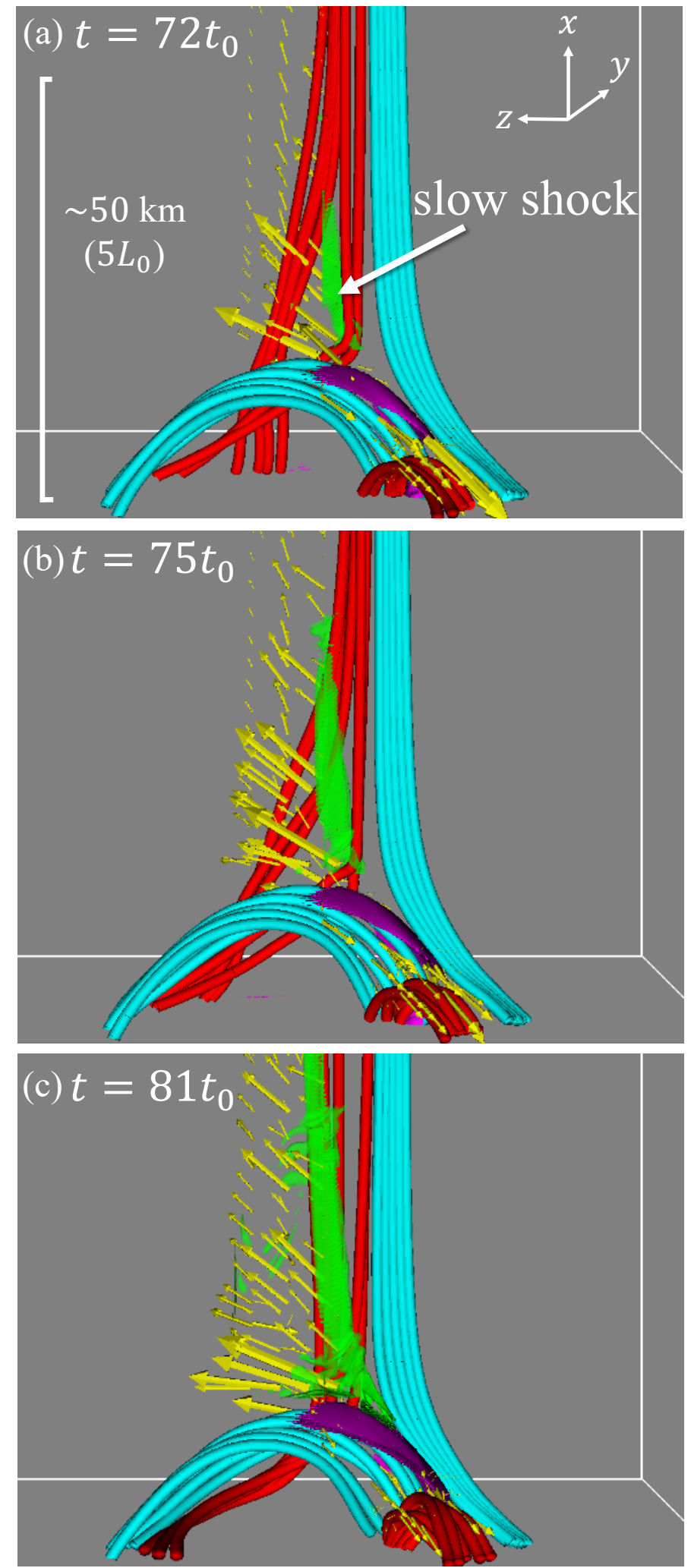

Fig. 12. 3D diagrams where the slow shock created by reconnection propagates in the direction of the background magnetic field. Green surface means $\operatorname{div} v=-0.09 / t_{0}$ and $-0.07 / t_{0}$, which corresponds to a slow shock created by reconnection. (a), (b), and (c) show $t=72 t_{0}, 75 t_{0}$, and $81 t_{0}$ cases. the color of magnetic field lines, the pink surface, and the yellow arrows indicate the same as figure 4. 
Alfvén waves released from the twisted sheared loop. In this case, the jets are accelerated by the magnetic pressure gradient. In our simulation, a twist of the reconnected field lines can be observed (figure 4) and Alfvén waves are generated (figure 14); however, they do not function well regarding the compression and acceleration of the plasma.

These differences from previous studies are due to our reconnection occurring in the region $\beta \sim 3-4$. In the $\beta \sim 3-4$ case, the reconnection outflow is subsonic; hence, a fast shock cannot be created where the outflow collides with the background field. Furthermore, because the magnetic energy is not dominant, it is difficult to accelerate the plasma using only the Lorentz force. However, a slow shock can be made if reconnection occurs.

There are several possible causes for the slow shock immediately propagating in the background direction. First, our reconnection outflow is subsonic; hence, it can bend before it collides with the background field. Secondly, the phase speed of the slow shock in the direction of the magnetic fields is Alfvén speed when $\beta \gg 1$. Therefore, the slow shock is unlikely to propagate in the outflow direction, unlike in the case of $\beta \ll 1$. Consequently, the slow shock is bent immediately after being formed on the current sheet and propagating in the background magnetic field (see figure 13).

Note that if these photospheric jet-like phenomena are observed, it is expected that the apparent speed obtained from the imaging observation and the line-of-sight speed obtained from the spectroscopic observation are different. In our simulation, the jet-like structure propagates at approximately $0.8 v_{0}=5.04 \mathrm{~km} \mathrm{~s}^{-1}$, corresponding to Alfvén speed. Conversely, the speed of the plasma accelerated by the slow shock is approximately $0.35 v_{0}=2.2 \mathrm{~km} \mathrm{~s}^{-1}$, which is approximately half the propagation speed of the slow shock.

\subsection{MHD wave propagation toward upper atmosphere}

To determine the energy flux of Alfvén wave $F_{\mathrm{A}}$, slow mode wave $F_{\text {slow }}$, and fast mode wave $F_{\text {fast }}$ are passing through the $x=$ const plane, we calculate these as follows:

$$
\begin{aligned}
& F_{\mathrm{A}}=\frac{1}{4 \pi} \frac{\int B_{0 x} \boldsymbol{v}_{\perp} \cdot \boldsymbol{\delta} \boldsymbol{B}_{\perp} d S}{\int d S} \\
& F_{\text {slow }}=\frac{\int \frac{B_{0 x}}{B_{0}} v_{\|} \delta p d S}{\int d S} \\
& F_{\text {fast }}=\frac{1}{4 \pi} \frac{\int B_{0 x} \delta B_{\|} v_{\perp} d S}{\int d S} \\
& F_{\text {kin }}=\frac{\int \rho v^{2} v_{x} d S}{\int d S} .
\end{aligned}
$$



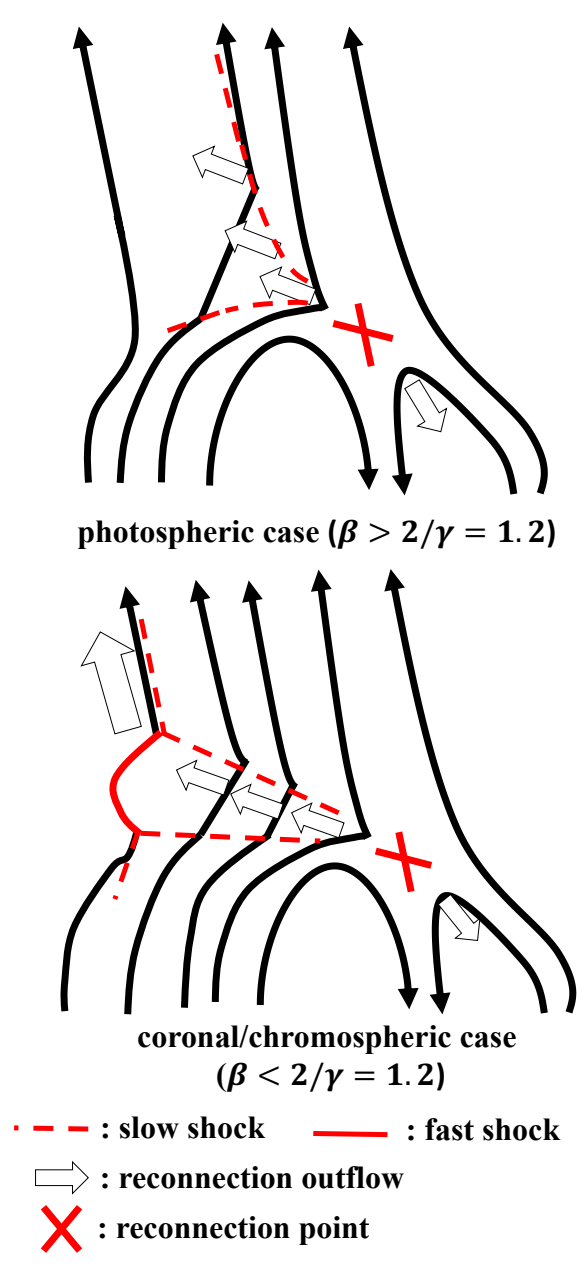

Fig. 13. Schematic diagrams of the difference in slow shock propagation direction due to the difference in plasma $\beta . \gamma=5 / 3$ is the specific heat ratio.

In the above equations, $\boldsymbol{\delta} \boldsymbol{B}=\boldsymbol{B}-\boldsymbol{B}_{0}$ and $\delta p=p-p_{0}$. The subscript $0, \perp$, and $\|$ are the values in the initial condition, direction normal, and parallel to the initial magnetic fields, respectively. $F_{\text {kin }}$ is the kinetic energy flux passing through the $x=$ const plane. We set an integration range as the region where the absolute value of each energy flux exceeds $25 \%$ of the maximum value of the absolute value of the energy flux. We measure these values in the $x=7.5 L_{0}, 15 L_{0}$, and $22.5 L_{0}$ planes, where plasma $\beta \simeq 2.0,1.25$, and 0.8. Note that equations (18) and (19) correspond to magnetosonic waves in the region where $\beta$ is less than one, and do not strictly correspond to the wave's energy flux. This is because the direction of the magnetosonic wave oscillation changes when $\beta$ is greater than one. However, regardless of the $\beta$ value, equations (17) and (18) correspond approximately to the components of the Poynting and enthalpy fluxes in the direction of the initial magnetic field, and equation (19) corresponds to the component normal to the initial field of the Poynting flux.

Figures 14a, 14c, and 14e display the result. At first, all mode waves are released, 
which corresponds to the sheared loop rising phase (figure $2 \mathrm{~b}$ ). The release of all modes in reconnection is the same as the results of Kigure et al. (2010). The reason for Alfvén wave dominance at $x=7.5 L_{0}$ is that the velocity in the $z$-direction, which is perpendicular to the initial background magnetic field, is the greatest. Subsequently, the twist of the sheared loop is released by magnetic reconnection, and the energy flux of the Alfvén mode becomes dominant in all planes. These features indicate that the jet-like structure is in Alfvénic motion, and also suggest that the reconnection process is similar to previous studies of coronal jets (e.g., Pariat et al. 2009; Archontis \& Hood 2013; Wyper et al. 2018). In each plane, all modes have approximately $10^{8} \mathrm{erg} \mathrm{cm}^{-2} \mathrm{~s}^{-1}$ in the $x=22.5 L_{0}$ plane. Moreover, the kinetic energy flux is smaller than the energy flux of the waves. This indicates that the nonlinearity of the plasma's motion forming the jet-like structure is not strong.

Figures 14b, 14d, and $14 \mathrm{f}$ display the mean value of the Mach number $M_{s}=v_{\|} / c_{s}$ and Alfvén Mach number $M_{A}=v_{\perp} / V_{A}$ in the area where slow and Alfvén mode is passing at each time. From these figures, we can observe that the peak value of each Mach number is approximately 0.1; this value is consistent with the case of the coronal jets (Yokoyama \& Shibata 1999). Furthermore, the Alfvén Mach number decreases with height, yet the Mach number of sound waves remains virtually unchanged. This is because, in our numerical settings, the Alfvén speed increases with height and the sound speed is uniform.

Figures 15, 16, and 17 display the energy flux of each wave and the distribution of the magnetic and gas pressures and $\operatorname{div} v$ in the $x=7.5 L_{0}$ and $x=15 L_{0}$ planes at $t=110 t_{0}$ and $x=22.5 L_{0}$ plane at $t=122 t_{0}$. From these figures, we can observe that the gas pressure increases and magnetic pressure decreases in the region of intense slow mode energy flux. Moreover, the jet-like structure can be observed to be in vortex motion. In figure 15, the region with strong wave energy flux is in virtually the same place, adjacent to the region with strong $\operatorname{div} v<0$. In figures 16 and 17, the regions with strong wave fluxes differ from each mode. In particular, it can be observed that the strong Alfvén wave region is where the magnetic pressure is strong, which is different from the region of the strong slow mode. Moreover, the strength of div $v$ is weaker than in the $x=7.5 L_{0}$ plane.

The reason the position of the peak of each mode is shifted as it rises is that the propagation speed of the slow mode and the Alfvén mode is different in the upper region. Figure 18 displays the 3D relationship between the $y z$ planes indicateing the distribution of the energy fluxes and magnetic field lines at $t=110 t_{0}$. Considering the vortex motion observable in figures 15,16 , and 17 , we can observe that a magnetic field line, along which waves propagate, moves in the order of the green, light blue, and red line with time. Figure 19 displays the time evolution 
of the distribution of energy fluxes along the magnetic field lines passing through a region with a strong slow mode in the $x=7.5 L_{0}$ plane. From this figure, we can observe that the positions of the peaks of the Alfvén mode and slow modes are virtually the same in the lower part, and that the Alfvén mode precedes in slow mode in the upper part. This is because where $\beta$ is greater than $2 / \gamma$, the slow mode propagates at the Alfvén velocity along the magnetic field line; however, below $2 / \gamma$, it propagates at the sound speed. From these facts, the position of the energy flux peaks appearing in the $y z$ cross section is different in the upper part because the slow mode passes after the Alfveń mode along the vortex-moving magnetic field lines.

We also investigated the effects of MHD waves generated from photospheric anemone jet-like structures on the upper atmosphere. From a simple estimate, we determined that the MHD wave's energy flux achieving a height of $500 \mathrm{~km}$ was approximately $4 \times 10^{7} \mathrm{erg} \mathrm{cm}^{-2} \mathrm{~s}^{-1}$ (see Appendix 1). This value is marginally greater than the amount required for chromospheric heating (Withbroe, \& Noyes 1977). Because of the small lifetime of the phenomenon, these waves are high frequency, and we can observe from figure 14 that the period is approximately $20 t_{0} \sim 30 \mathrm{~s}$. This implies that the photospheric anemone jet-like structure could be one of the origins of the high-frequency Alfvén waves that have been observed in the spicules (He et al. 2009; Okamoto \& De Pontieu 2011), though other candidates exist, such as mode conversions of longitudinal to transverse waves (Shoda \& Yokoyama 2018). Furthermore, we extended the computational domain to the corona and performed 1D hydrodynamic simulations. From the results of the simulations, we determined that the photospheric jet-like structure can also influence the spicule formation (see Appendix 2). Note that these estimates are simplistic and should be studied in more detail in future papers.

\section{Conclusion}

We performed 3D MHD simulation of anemone jet-like structures in the solar photospheric parameter. From the results of the simulation, a jet-like structure was induced by magnetic reconnection. The length, width, lifetime, and apparent velocity of the jet-like structure were extensions of the coronal and chromospheric anemone jets. This jet-like structure was formed by the propagation of the slow shock generated by magnetic reconnection. These facts indicate that the anemone jet-like structure, which can be explained by the unified model (Shibata 1999; Shibata et al. 2007), is expected to exist in the solar photosphere; however, the formation process of the jet-like structure is different from that in the low $\beta$ environment of the chromosphere and corona. In the present study, we simulated a jet-like structure with a length of approximately 
flux $\left(10^{8} \mathrm{erg} \mathrm{cm}^{-2} \mathrm{~s}^{-1}\right)$
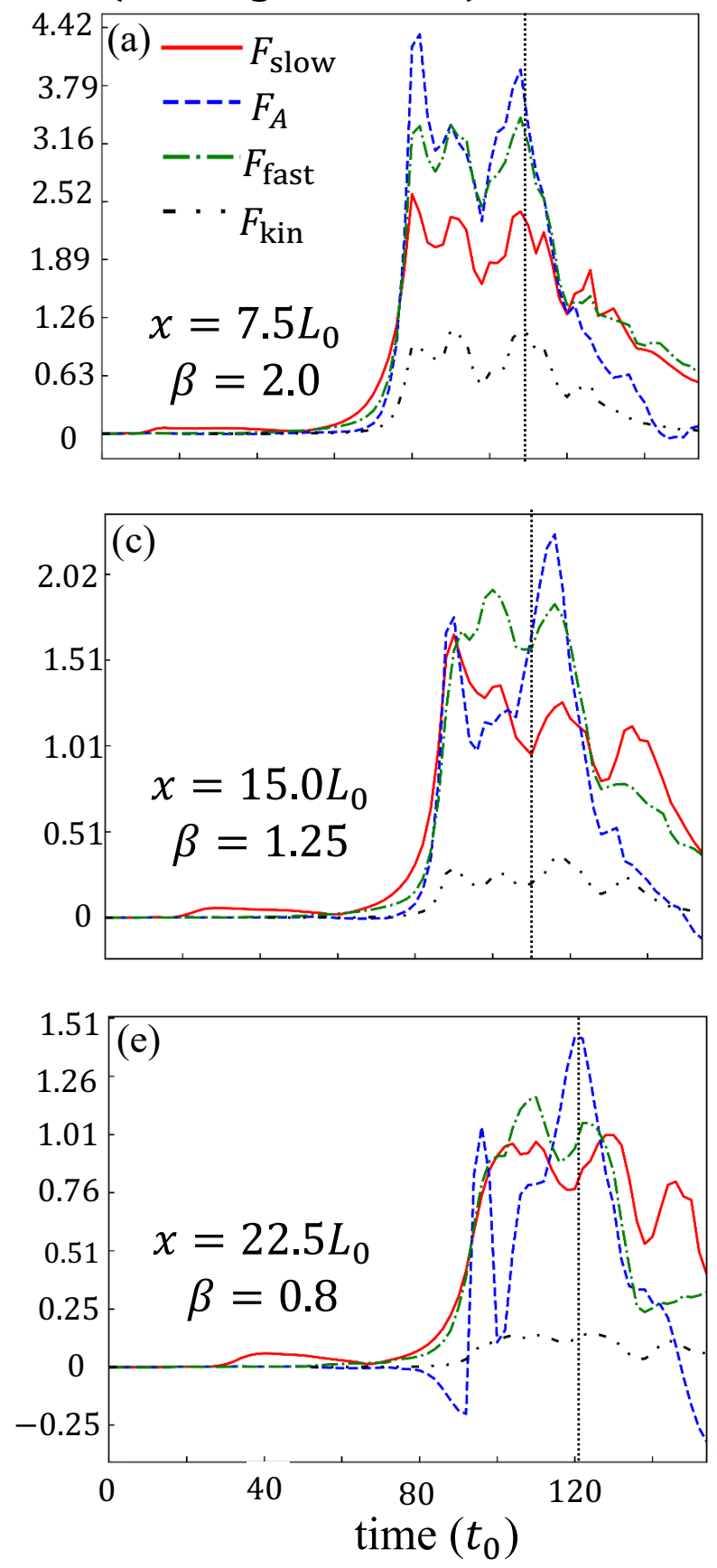

\section{dimensionless values}
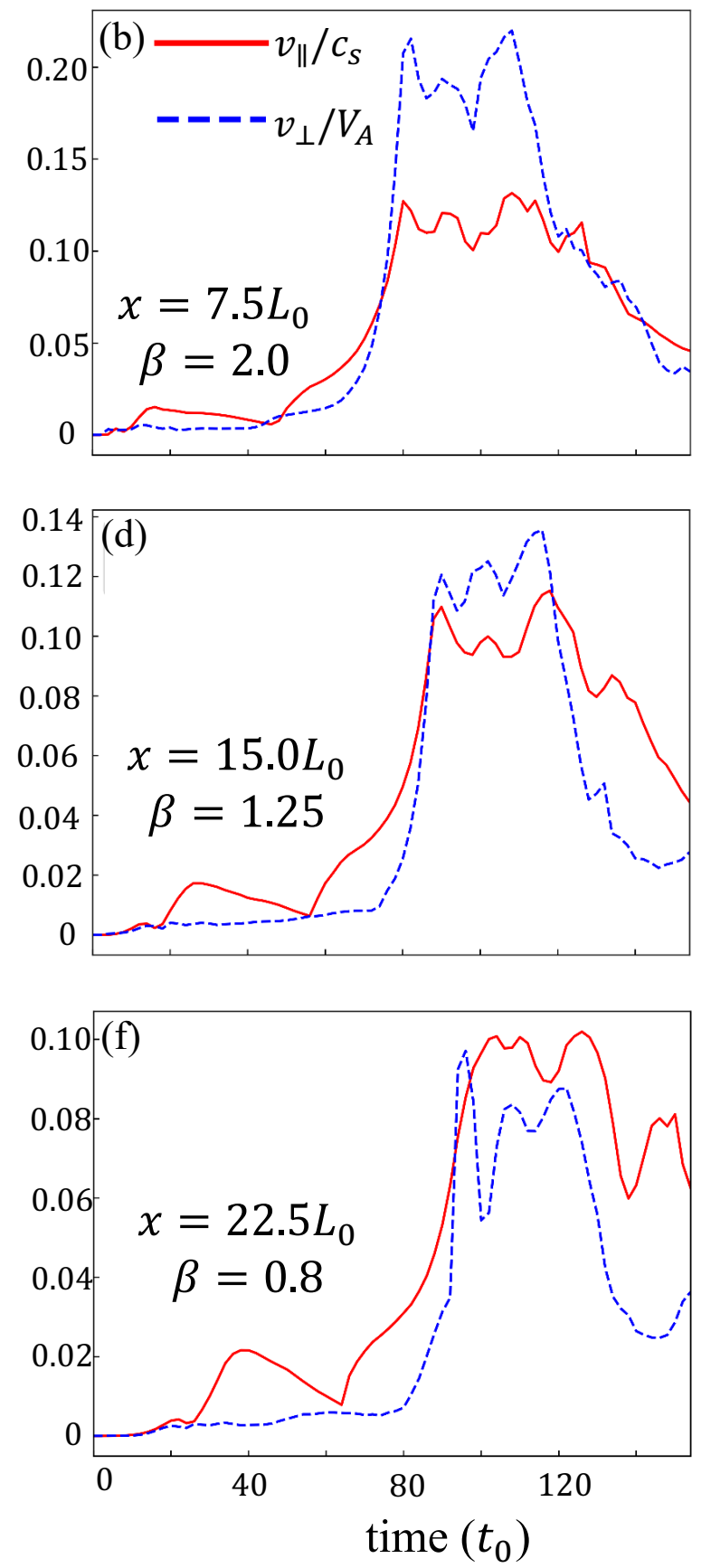

Fig. 14. (a), (c), (e): Time development of MHD wave energy flux passing through $x=7.5 L_{0}, 15 L_{0}$, and $22.5 L_{0}$ plane. Dashed blue, solid red, and dash-dot green lines show Alfvén, slow, and fast mode. Dash-dot-dot black lines indicate kinematic energy flux. The dotted line shown in (a) and (c) indicates $t=110 t_{0}$, the time shown in figures 15 and 16. The dotted line indicated by (e) denotes $t=122 t_{0}$, which is the time indicated in figure 17. (b), (d), (f): Time development of Mach number of Alfvén and sound wave. Blue dashed and red solid lines show Alfvén and sound waves. 

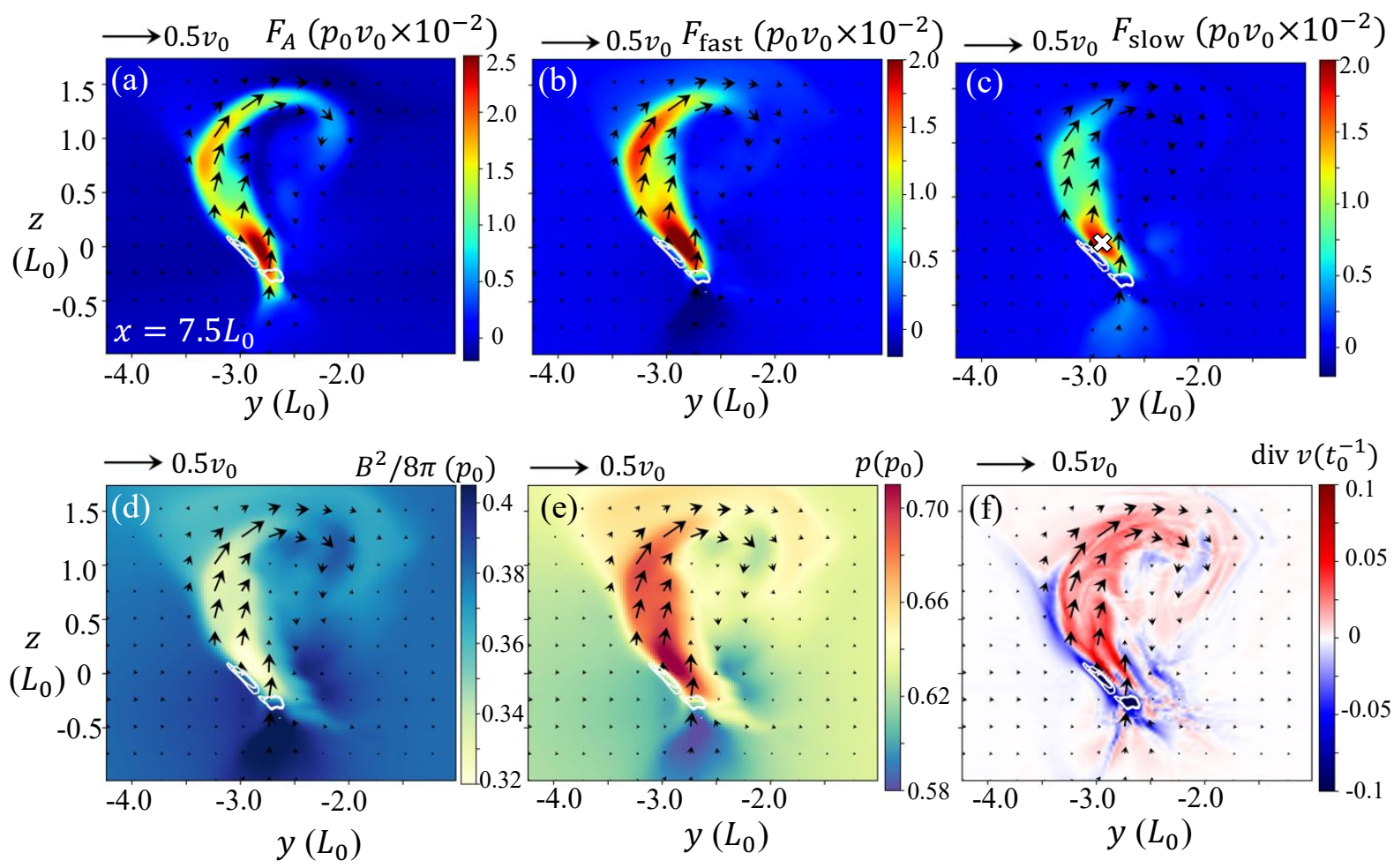

Fig. 15. Energy flux of each wave and the distribution of magnetic and gas pressures and $\operatorname{div} v$ in the $x=7.5 L_{0}$ plane at $t=110 t_{0}$. Black arrows show velocity in the plane. White contours indicate $\operatorname{div} v=-0.07 t_{0}^{-1}$ and $-0.09 t_{0}^{-1}$. The white $\mathrm{x}$ mark in (c) indicates the area where the magnetic field line is passing through in figure 18.

$100 \mathrm{~km}$. However, such a jet-like structure is expected to be formed in an environment where the plasma $\beta$ is greater than one, even though the scales are different.

We also confirmed, for the first time, that the magnetosonic and Alfvén waves are generated by magnetic reconnection in the solar photospheric parameters in a $3 \mathrm{D}$ manner . These waves are high frequency, and their non-dimensional amplitudes are comparable with those of the coronal case. Furthermore, the MHD wave energy fluxes were generated to the degree that could influence the local chromospheric heating and the formation of spicules.

\section{Acknowledgments}

We would like to Mr. T. Sakaue for his useful comments regarding the proposed numerical simulation and interpreting the simulation results. We also thank Dr. T. Yokoyama and Dr. S. Toriumi for their fruitful discussions. We are deeply grateful to Dr. K. Tomida for instructing us on the use of Athena++. Furthermore, we wish to thank the anonymous referee for helpful comments that led to improvements in this work. We would like to thank Editage (www.editage.com) for English language editing. Numerical computations were carried out on Cray XC50 at Center for Computational Astrophysics, National Astronomical Observatory of Japan. 

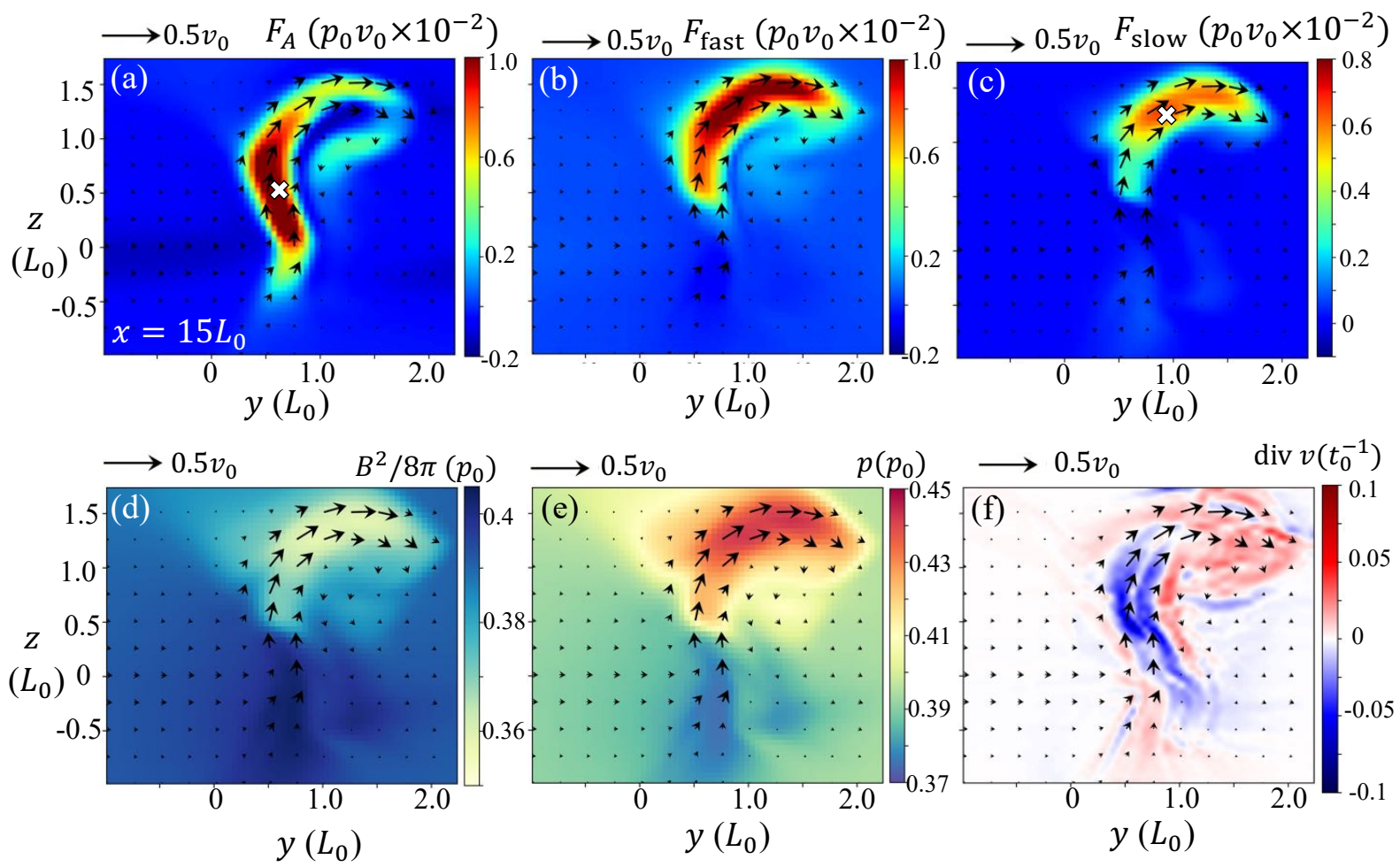

Fig. 16. Energy flux of each wave and the distribution of magnetic and gas pressures and $\operatorname{div} v$ in the $x=15 L_{0}$ plane at $t=110 t_{0}$. Black arrows show velocity in the plane. The white $x$ mark in (c) indicates the area where the magnetic field line is passing through in figure 18.

\section{Supporting Information}

The following Supporting Information is available in the online version of this article. E-movies 1 and 2 .

\section{Appendix 1 Effect of chromospheric heating}

Figure 14e indicates that each mode has approximately $10^{8} \mathrm{erg} \mathrm{cm}^{-2} \mathrm{~s}^{-1}$ in the $x=22.5 L_{0}=$ $225 \mathrm{~km}$ plane. To discuss the effect of chromospheric heating, we estimate the amount of energy flux that can reach the chromosphere at a height of $\sim 500 \mathrm{~km}$ as follows. We consider an Alfvén wave and slow mode wave propagating along a vertical magnetic flux tube with cross section $S$. First, we assume a pressure balance between the inside and outside flux tube, $B^{2} / 8 \pi \sim p \propto e^{-x / H}$; then, $B \propto e^{-x / 2 H}$. Secondly, we assume a magnetic flux conservation $B S=$ const; then, $S \propto e^{x / 2 H}$. Finally, we assume energy conservation $F S=$ const, where $F$ is the energy flux. Considering that a wave flux passing through the $x=225 \mathrm{~km}$ plane $F_{x=225 \mathrm{~km}}$ is approximately $10^{8} \mathrm{erg} \mathrm{cm}^{-2} \mathrm{~s}^{-1}$, then a wave flux passing through the $x=500 \mathrm{~km}$ plane $F_{x=500 \mathrm{~km}}$ can be estimated. 

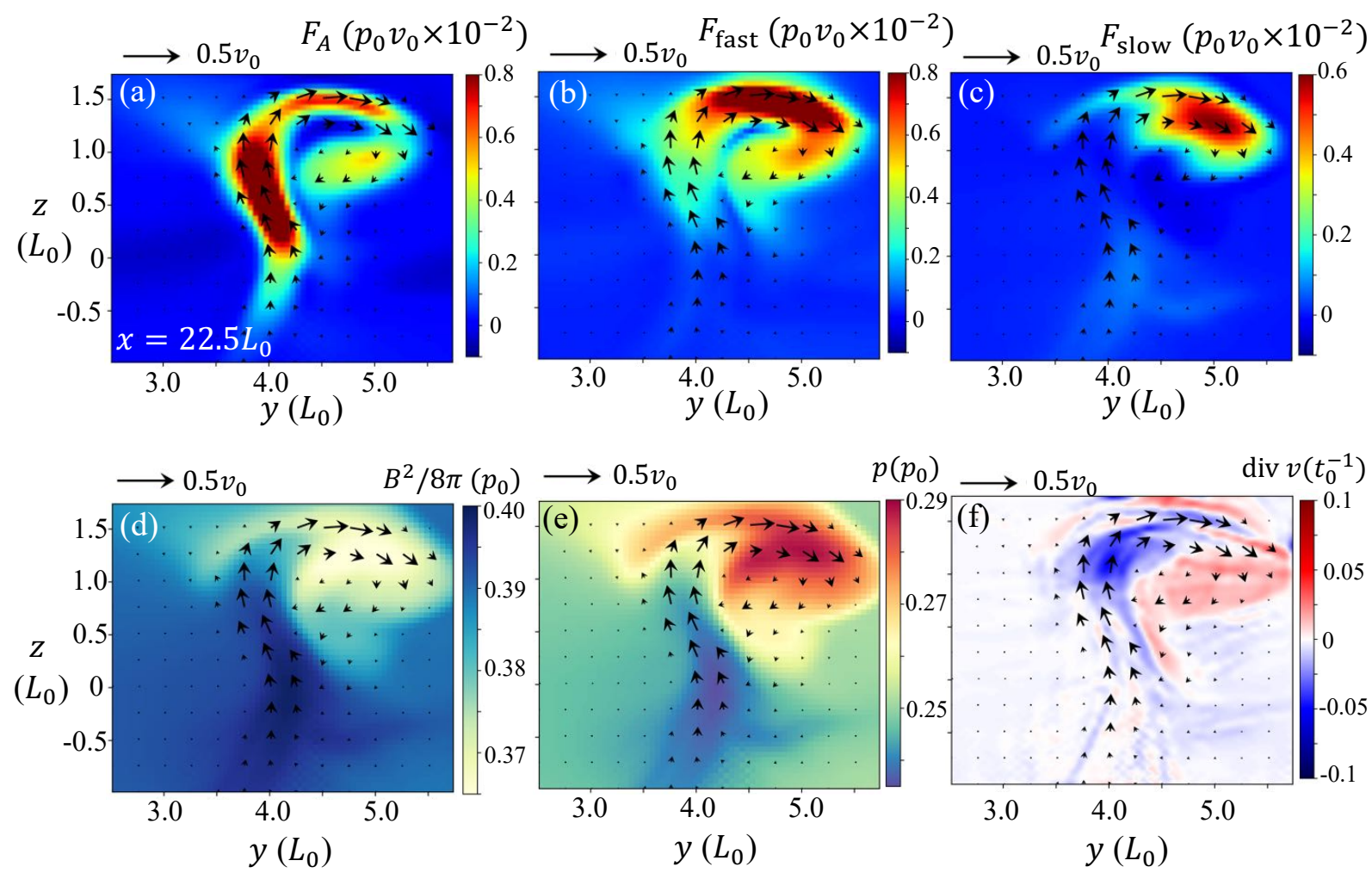

Fig. 17. Energy flux of each wave and the distribution of magnetic and gas pressures and $\operatorname{div} v$ in the $x=22.5 L_{0}$ plane at $t=122 t_{0}$. Black arrows show velocity in the plane.

$$
\begin{array}{r}
F_{x=500 \mathrm{~km}} \sim F_{x=225 \mathrm{~km}} \times e^{(225 \mathrm{~km}-500 \mathrm{~km}) / 2 H} \\
\sim 4 \times 10^{7} \mathrm{erg} \mathrm{cm}^{-2} \mathrm{~s}^{-1}
\end{array}
$$

This value is approximately the energy flux required for heating the chromosphere in the active region $1.5 \times 10^{7} \mathrm{ergcm}^{-2} \mathrm{~s}^{-1}$ and greater than $4 \times 10^{6} \mathrm{ergcm}^{-2} \mathrm{~s}^{-1}$ in the quiet region (Withbroe, \& Noyes 1977). Because these waves are high frequency, the transverse waves are easily converted to longitudinal waves by mode conversion, and the majority of these are dissipated in the chromosphere (Matsumoto \& Shibata 2010). Assuming the loop-like geometry found in the active regions, Antolin \& Shibata (2010) found that active regions may not be heated by Alfvén waves, based on the expansion factor of the loop and other observed facts. Therefore, the waves released from the photospheric anemone jet-like structure are expected to contribute mainly to the heating of the chromosphere. Note that we must consider the frequency of the jet-like structure to discuss the contribution to global chromospheric heating because these energy fluxes originate from a photospheric jet-like structure. 

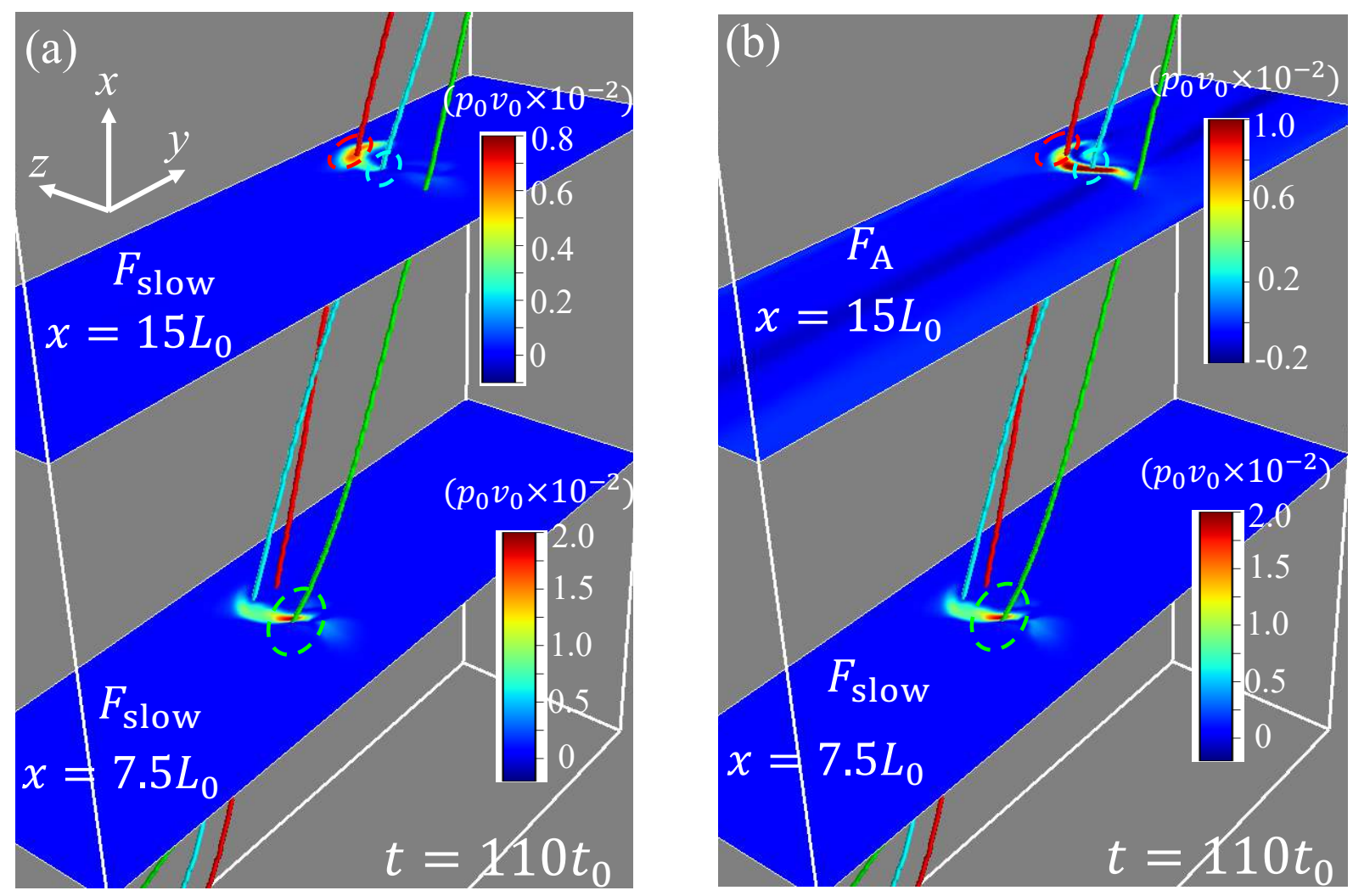

Fig. 18. 3D relationship between the $y z$ plane showing the distribution of energy fluxes and magnetic field lines at $t=110 t_{0}$. In (a), we show the distribution of slow mode energy flux in the $x=15 L_{0}$ plane shown in figure 16c, and in (b), we show that of Alfvén mode shown in figure 16a. In both figures, the $x=7.5 L_{0}$ plane shows the distribution of slow mode energy flux shown in figure $15 \mathrm{c}$. The green line indicates a magnetic field line passing through a region with a strong energy flux in the $x=7.5 L_{0}$ plane. The light blue line shows a magnetic field line passing through a region with a strong energy flux of Alfvén mode in the $x=15 L_{0}$ plane, and the red linesshow a magnetic field line passing through a region with a strong energy flux of slow mode in the same plane. The three dashed lines surrounding the magnetic field lines indicate the region through which each magnetic field line passes.

\section{Appendix 2 1D simulation for the spicule formation}

To investigate how slow mode waves generated from the photospheric anemone jet-like structures propagate to the upper atmosphere along a vertical magnetic flux tube, we perform 1D non-magnetic hydrodynamic simulations.

For the numerical simulation, we use Athena++ code with the van Leer predictorcorrector scheme and Piecewise Linear Method (Stone et al. 2020). We solve the compressive hydrodynamic equation including uniform gravity. The basic equations are as follows.

$$
\begin{aligned}
& \frac{\partial \rho}{\partial t}+\frac{\partial}{\partial s}\left(\rho v_{\|}\right)=0 \\
& \frac{\partial \rho v_{\|}}{\partial t}+\frac{\partial}{\partial s}\left(\rho v_{\|}^{2}\right)=-\frac{\partial}{\partial s} p+\rho g_{\|} \\
& \frac{\partial}{\partial t}\left(e+\frac{1}{2} \rho v_{\|}^{2}\right)+\frac{\partial}{\partial s}\left[\left(h+\frac{1}{2} \rho v_{\|}^{2}\right) v_{\|}\right]=\rho g_{\|} v_{\|}
\end{aligned}
$$




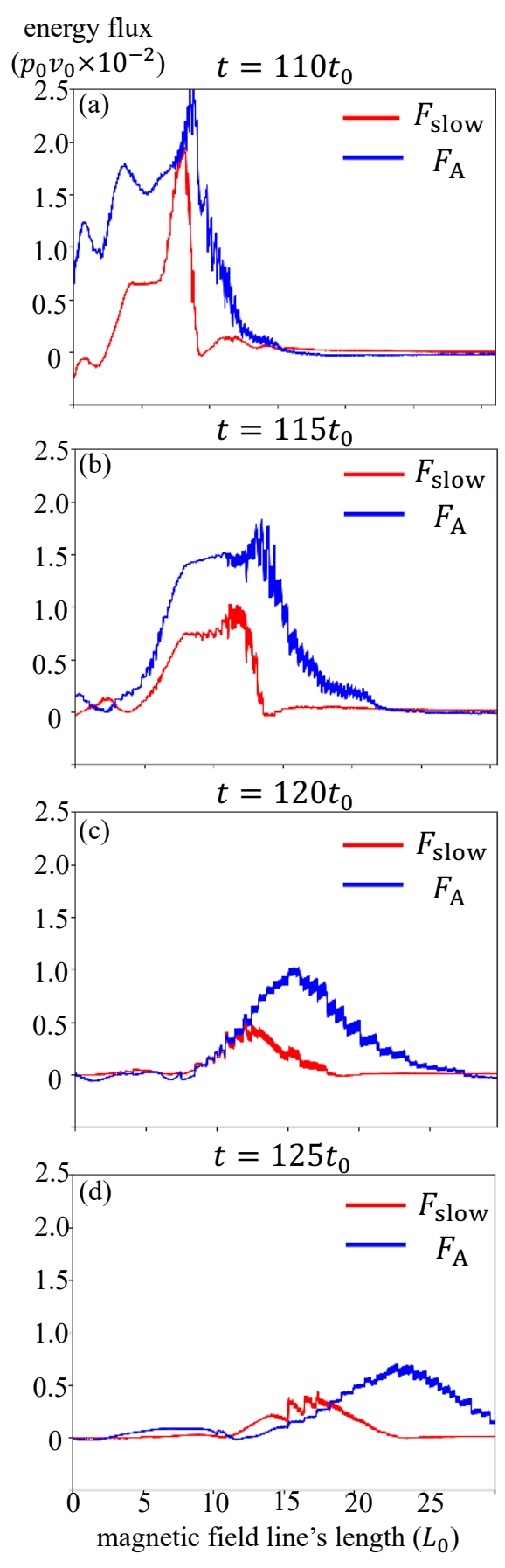

Fig. 19. Time evolution of the distribution of energy fluxes along the green magnetic field line shown in figure 18 , which is passing through a region with a strong slow mode in the $x=7.5 L_{0}$ plane. The time evolution is calculated by tracking the magnetic field line in a Lagrangian way. The horizontal axis shows the length of the magnetic field line when the bottom surface is set to zero. The red and blue lines show the slow and Alfvén mode energy fluxes, respectively. (a), (b), (c), and (d) show the distribution at $t=110 t_{0}, 115 t_{0}, 120 t_{0}$, and $125 t_{0}$, respectively. 


$$
p=\frac{\rho R T}{\mu}
$$

, where $e$ is the internal fluid energy and $h=p+e$ is the enthalpy.

For the coordinates of the 1D calculation, a parallel straight line is taken in the background field of the 3D calculation. We set the height of the upper boundary as $12,000 \mathrm{~km}$ from the bottom of the photosphere. The number of the mesh is 24,000, and the grid spacing is uniform. The normalization of the numerical calculation is performed in the same manner as the 3D calculation. We use only the background field component for the gravitational acceleration.

As the initial condition, we assume hydrostatic equilibrium with initial temperature $T_{\text {ini }}$.

$$
T_{\text {ini }}=T_{\text {pho }}+\frac{1}{2}\left(T_{\text {cor }}-T_{\text {pho }}\right)\left(1+\tanh \left(\frac{x-x_{\text {tr }}}{w_{\text {tr }}}\right)\right)
$$

$T_{\mathrm{pho}}, T_{\mathrm{cor}}, x_{\mathrm{tr}}$, and $w_{\mathrm{tr}}$ are the temperature of the photosphere and corona, and the height and the thickness of the transition layer. We set $T_{\text {pho }}=6000 \mathrm{~K}, w_{\mathrm{tr}}=80 \mathrm{~km}$. Then, along a reconnected field line in 3D simulation, we take out the thermodynamic quantity and velocity components parallel to the field and consider them as a perturbation. For the parameters $T_{\text {cor }}$ and $x_{\mathrm{tr}}$, we perform two cases, $T_{\text {cor }}=170 T_{\mathrm{pho}}=1.02 \times 10^{6} \mathrm{~K}, x_{\mathrm{tr}}=2300 \mathrm{~km}$ (quiet region case) and $T_{\text {cor }}=400 T_{\text {pho }}=2.4 \times 10^{6} \mathrm{~K}, x_{\mathrm{tr}}=1850 \mathrm{~km}$ (active region case). The coronal pressure in the initial condition is a approximate agreement with the values reported in the observations (quiet region: Ito et al. 2010, active region: Winebarger et al. 2011).

For the boundary condition, we set the reflected boundary at the bottom and open boundary at the top. Note that in order to maintain the hydrostatic pressure equilibrium at the upper boundary, we set the gravitational acceleration to zero smoothly above the height of $11,000 \mathrm{~km}$. This height is sufficiently greater than that at which the contact discontinuity surface obtained from the calculation results rises, and thus this assumption does not substantially influence the calculation.

Figure 20 displays the results. We can observe that the contact discontinuity is launched by shocks. Figures 20a and 20b display the quiet region case, and we can observe that the maximum height is approximately $3200 \mathrm{~km}$, the maximum velocity is approximately $20 \mathrm{kms}^{-1}$, and the lifetime is approximately $160 \mathrm{~s}$. Pereira et al. (2012) and Zhang et al. (2012) performed a statistical study of spicules, and many of their results are consistent with our simulation. Figures 20c and 20d display the active region case. We can observe that the maximum height is approximately $2100 \mathrm{~km}$ (the maximum length is approximately $300 \mathrm{~km}$ ), the maximum velocity is approximately $15 \mathrm{kms}^{-1}$, and the lifetime is approximately $100 \mathrm{~s}$. De Pontieu et al. (2007) and Anan et al. (2010) performed statistical studies of dynamic fibrils. Their studies 


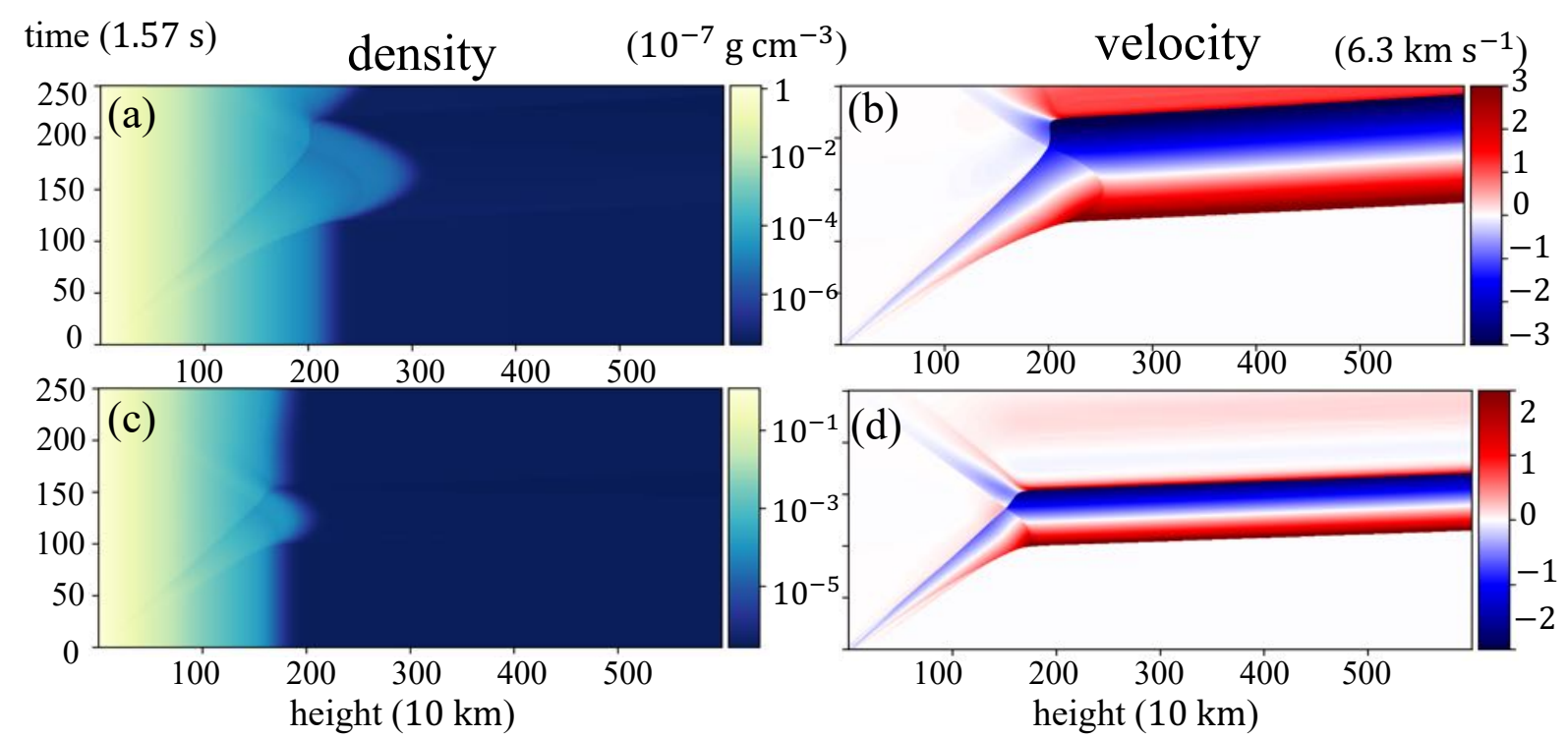

Fig. 20. Results of 1D simulation. (a), (c): time-height plot of density. (b), (d): time-height plot of velocity. (a), (b) and (c), (d) correspond to quiet region and active region cases.

are consistent with our results regarding the maximum length and the maximum velocity. The maximum length and lifetime in our results are marginally shorter than their study, yet reasonably consistent with theirs. These results suggest that photospheric anemone jet-like structures can be one of the origins of spicules and dynamic fibrils.

Figure 21 displays the trajectories of the fluid particles. From this figure, we can observe that the fluid particles do not move to a great degree in the lower layer, yet near the transition region, they are significantly launched by the shock to form a jet. Note that the behavior of the fluid particles in the lower layers in this figure is similar to that of those observable in figure 9. This is consistent with the result that the photospheric anemone jet-like structure is not a plasma flow.

\section{References}

Anan, T., Kitai, R., Kawate, T., et al. 2010, PASJ, 62, 871

Antolin, P., \& Shibata, K. 2010, ApJ, 712, 494

Archontis, V., \& Hood, A. W. 2013, ApJL, 769, L21

Athay, R. G. 1976, Astrophysics and Space Science Library, Vol. 53, The solar chromosphere and corona: Quiet sun, Dordrecht: Reidel, 474

Bharti, L., Solanki, S. K., \& Hirzberger, J. 2017, A\&A, 597, A127

Chitta, L. P., Peter, H., Solanki, S. K., et al. 2017, ApJS, 229, 4 


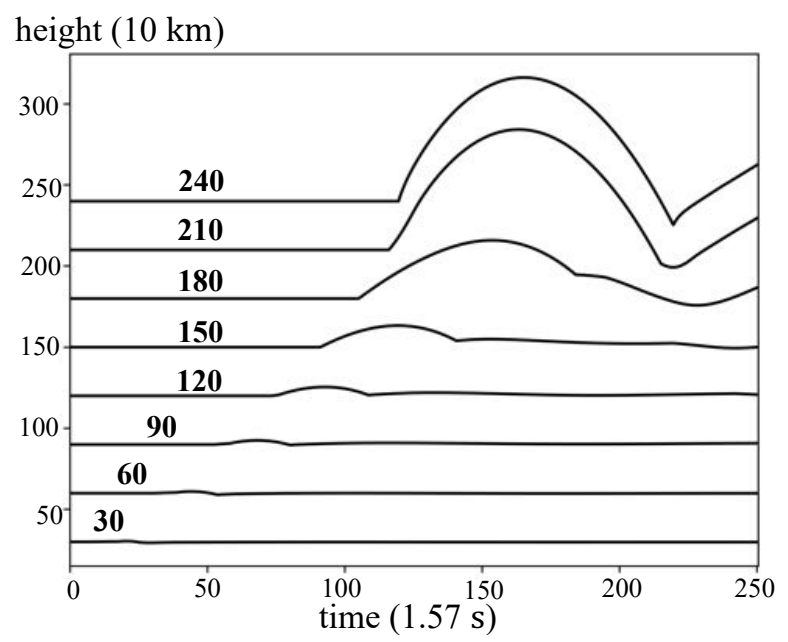

Fig. 21. Trajectories of the fluid particles in the case of the quiet region. The number above each curve indicates the initial position of each fluid particle.

De Pontieu, B., Erdélyi, R., \& James, S. P. 2004, Nature, 430, 536

De Pontieu, B., Hansteen, V. H., Rouppe van der Voort, L., et al. 2007, ApJ, 655, 624

Fang, F., Fan, Y., \& McIntosh, S. W. 2014, ApJL, 789, L19

He, J.-S., Tu, C.-Y., Marsch, E., et al. 2009, A\&A, 497, 525

Hollweg, J. V., Jackson, S., \& Galloway, D. 1982, Sol. Phys., 75, 35

Ito, H., Tsuneta, S., Shiota, D., et al. 2010, ApJ, 719, 131

Kigure, H., Takahashi, K., Shibata, K., et al. 2010, PASJ, 62, 993

Kudoh, T., \& Shibata, K. 1999, ApJ, 514, 493

Leake, J. E., \& Arber, T. D. 2006, A\&A, 450, 805

Lee, E. J., Archontis, V., \& Hood, A. W. 2015, ApJL, 798, L10

Matsumoto, T., \& Shibata, K. 2010, ApJ, 710, 1857

Moreno-Insertis, F., Galsgaard, K., \& Ugarte-Urra, I. 2008, ApJL, 673, L211

Moreno-Insertis, F., \& Galsgaard, K. 2013, ApJ, 771, 20

Nelson, C. J., Freij, N., Bennett, S., et al. 2019, ApJ, 883, 115

Nisticò, G., Bothmer, V., Patsourakos, S., et al. 2009, Sol. Phys., 259, 87

Nishizuka, N., Shimizu, M., Nakamura, T., et al. 2008, ApJL, 683, L83

Okamoto, T. J., \& De Pontieu, B. 2011, ApJL, 736, L24

Pariat, E., Antiochos, S. K., \& DeVore, C. R. 2009, ApJ, 691, 61

Pariat, E., Dalmasse, K., DeVore, C. R., Antiochos, S. K., \& Karpen, J. T. 2015, A\&A, 573, A130

Pariat, E., Dalmasse, K., DeVore, C. R., Antiochos, S. K., \& Karpen, J. T. 2016, A\&A, 596, A36

Parker, E. N. 1991, ApJ, 372, 719

Pereira, T. M. D., De Pontieu, B., \& Carlsson, M. 2012, ApJ, 759, 18 
Petschek, H. E. 1964, NASA Special Publication, 425

Shibata, K., Ishido, Y., Acton, L. W., et al. 1992, PASJ, 44, L173

Shibata, K., Nitta, N., Strong, K. T., et al. 1994, ApJL, 431, L51

Shibata, K. 1999, Ap\&SS, 264, 129

Shibata, K., Nakamura, T., Matsumoto, T., et al. 2007, Science, 318, 1591

Shimojo, M., Hashimoto, S., Shibata, K., et al. 1996, PASJ, 48, 123

Shoda, M., \& Yokoyama, T. 2018, ApJ, 854, 9

Singh, K. A. P., Shibata, K., Nishizuka, N., \& Isobe, H. 2011, Physics of Plasmas, 18, 111210

Spitzer, L. 1962, Physics of Fully Ionized Gases

Sterling, A. C., \& Moore, R. L. 2016, ApJL, 828, L9

Stone, J. M., Tomida, K., White, C. J., et al. 2020, arXiv e-prints, arXiv:2005.06651

Suematsu, Y., Shibata, K., Neshikawa, T., \& Kitai, R. 1982, Sol. Phys., 75, 99

Takasao, S., Isobe, H., \& Shibata, K. 2013, PASJ, 65, 62

Török, T., Aulanier, G., Schmieder, B., Reeves, K. K., \& Golub, L. 2009, ApJ, 704, 485

Tian, H., Yurchyshyn, V., Peter, H., et al. 2018, ApJ, 854, 92

Wang, Y., \& Yokoyama, T. 2020, ApJ, 891, 110

Winebarger, A. R., Schmelz, J. T., Warren, H. P., et al. 2011, ApJ, 740, 2

Withbroe, G. L., \& Noyes, R. W. 1977, ARA\&A, 15, 363

Wyper, P. F., DeVore, C. R., \& Antiochos, S. K. 2018, ApJ, 852, 98

Yang, H., Chae, J., Lim, E.-K., et al. 2014, ApJL, 790, L4

Yurchyshyn, V. B., Goode, P. R., Abramenko, V. I., \& Steiner, O. 2011, ApJL, 736, L35

Yokoyama, T., \& Shibata, K. 1996, PASJ, 48, 353

Yokoyama, T., \& Shibata, K. 1999, American Institute of Physics Conference Series, 61

Zhang, Y. Z., Shibata, K., Wang, J. X., et al. 2012, ApJ, 750, 16 